COMMUNICATIONS IN

ANALYSIS AND GEOMETRY

Volume 6, Number 4, 705-747, 1998

\title{
Deformations of Calibrated Submanifolds
}

\author{
ROBERT C. MCLEAN
}

\begin{abstract}
Assuming the ambient manifold is Kähler, the theory of complex submanifolds can be placed in the more general context of calibrated submanifolds, see [HL]. It is therefore natural to try to extend some of the many results in complex geometry to the other calibrated geometries of [HL]. In particular, the question of deformability of calibrated submanifolds is addressed here (analogous to Kodaira's work on deformations of complex submanifolds [K]). Also, a formula for the second variation of volume of an arbitrary calibrated submanifolds which generalizes a result of Simons' in the complex category $[\mathrm{S}]$ is given.
\end{abstract}

\section{Introduction and summary.}

General Remarks. In this paper, we discuss the deformation theory of calibrated submanifolds of Riemannian manifolds with restricted holonomy. Most of the definitions of the terms used in this introduction can be found in the seminal paper of Harvey and Lawson ([HL]). Throughout, we denote the ambient manifold by $M$ and the submanifold by $X$.

Recall that a calibration is a closed $p$-form $\varphi$ on a Riemannian manifold $M^{n}$ such that $\varphi$ has comass 1, i.e., $\varphi$ restricts to each tangent $p$-plane of $M$ to be less than or equal to the volume form of that $p$-plane. The form $\varphi$ then singles out those submanifolds for which there is equality, that is, those submanifolds for which $\varphi$ restricts to be equal to the Riemannian volume form. Such submanifolds are said to be calibrated by the form $\varphi$. It is easily shown that a calibrated submanifold has least volume in its homology class. In particular, calibrated submanifolds are stable minimal submanifolds. In this paper, we use the term calibrated geometry for the ambient manifold $M$, the calibration $\varphi$, and the collection of submanifolds calibrated by $\varphi$.

The primary example of a calibrated geometry is when the ambient manifold $\mathrm{M}$ has holonomy contained in $U(n)$, that is to say, it is a Kähler manifold. If $\kappa$ denotes the Kähler form and if $\varphi_{p}=\frac{1}{p !} \kappa^{p}$, then $\varphi_{p}$ satisfies the conditions to be a calibration: By the Kähler assumption, $\varphi_{p}$ is closed, 
and by the classical Wirtinger's inequality (see [HL]), $\varphi_{p}$ restricts to be less than or equal to the volume form on any tangent $2 p$-plane. Furthermore, the real $2 p$-planes for which the $\varphi_{p}$ is the volume form are the complex $p$ planes. Hence, the submanifolds calibrated by $\varphi_{p}$ are precisely the complex $p$-dimensional submanifolds. Hence complex submanifolds are volume minimizing in their homology classes. This latter observation is originally due to Federer.

In [HL], Harvey and Lawson give four new examples of calibrated geometries. The first is the special Lagrangian calibration. This is a real $n$-form defined on a $2 n$-dimensional manifold with holonomy contained in $S U(n)$. The other three are exceptional calibrations in the sense that they only occur in specific dimensions. They are: the associative calibration, a 3 -form defined on a seven dimensional manifold with holonomy contained in $G_{2}$; the coassociative calibration, a 4-form also defined on seven dimensional manifold with holonomy contained in $G_{2}$; and lastly, the Cayley calibration, which is a 4-form defined on eight dimensional manifolds with holonomy contained in Spin(7). We will give the precise definitions of these forms in the appropriate sections.

Since complex submanifolds can be placed in the more general context of calibrated submanifolds (assuming the Kählerness), it is natural to try to generalize results in complex geometry to the other calibrated geometries. This is what is done here. The rub is that we must restrict ourselves to generalizing results in the the differential geometry of complex submanifolds, eschewing results only provable with algebraic geometric techniques. With complex geometry in mind, a natural subject is the deformability of calibrated submanifolds.

The literature on the deformation and moduli theory of complex submanifolds is extensive, but let us recall some key results. One first linearizes the problem of finding deformations of a given complex submanifold $X$, i.e., one finds deformations of $X$ up to first order. The space of such first order deformations is called the Zariski tangent space to the moduli space of complex submanifolds. It is easy to see that these first order deformations merely correspond to holomorphic sections of the normal bundle of X. That is to say, the Zariski tangent space is $\check{H}^{0}\left(\mathcal{N}_{X}\right)$ (Čech cohomology with values in in the sheaf associated with the normal bundle $N(X))$.

The first key result of deformation theory is the following: In $[\mathrm{K}]$, Kodaira shows that if $\breve{H}^{1}\left(\mathcal{N}_{X}\right)=0$, then, given a first order deformation of $X$, this first order deformation is realized by an actual deformation. More generally, given a deformation to first order (resp., $m^{\text {th }}$ order), then there is an obstruction, lying in $\breve{H}^{1}\left(\mathcal{N}_{X}\right)$, to extending it to a deformation to second 
(resp., $\left.(m+1)^{\text {st }}\right)$ order. Hence, if $\check{H}^{1}\left(\mathcal{N}_{X}\right)=0$, then all obstructions vanish automatically.

There is a particular case that will have analogs in what follows. If one looks at smooth complex hypersurfaces $X$ of a complex manifold $M$ with canonical bundle $K_{M}$ trivial, then by the adjunction formula ([GH], pp. 147)

$$
N(X)=\left.K_{M}\right|_{X} \otimes N(X)=K_{X} .
$$

Thus, the normal bundle is actually intrinsic, and we may rephrase deformation questions about $X$ in $M$ intrinsically. For example, if the geometric genus $p_{g}(X)=\operatorname{dim} \check{H}^{0}\left(K_{X}\right)$ is zero, then $X$ will be rigid as a complex submanifold.

In algebraic geometry, one can put various structures on the moduli space depending on the category in which one is working. In particular in the algebraic category, Grothendieck [G] has shown that one can put a scheme structure, the Hilbert scheme, on the moduli space $\mathcal{M}$ of algebraic subvarieties. At unobstructed point $X$ of $\mathcal{M}$, the tangent space to the $\mathcal{M}$ is precisely $\breve{H}^{0}\left(\mathcal{N}_{X}\right)$. (If one is uncomfortable with schemes, one may think of the moduli space of complex submanifolds as just a complex variety, possibly with singularities and multiplicities.)

Lastly, we recall a result of Simons (see $[\mathrm{S}]$ or $[\mathrm{L}]$ ) that computes the second variation of volume for a complex submanifold $X$ of a Kähler manifold $M$. Let $X_{t}, t \in(-\epsilon, \epsilon)$ be a normal deformation to $X=X_{0}$ (i.e., the deformation vector field $V=\frac{\partial}{\partial t} X_{t}$ is normal to $X_{t}$.) Then Simons' formula is:

$$
\left.\frac{d^{2}}{d t^{2}} \operatorname{vol}\left(X_{t}\right)\right|_{t=0}=\frac{1}{2} \int_{X}\|\bar{\partial}(V)\|^{2} d \operatorname{vol}_{X} .
$$

This simple expression should be contrasted with the usual expression of the second variation of volume involving both the curvature of the ambient manifold and also the second fundamental form of the immersion, see [Ch1] or $[\mathrm{L}]$. This gives an infinitesimal proof of the stability of complex submanifolds as minimal submanifolds. Also, one sees that the only way to deform a complex submanifold through minimal submanifolds is to deform it through complex submanifolds.

In this paper, we first restrict ourselves to two particular calibrated geometries, namely, the special Lagrangian and coassociative geometries. In these cases, the normal bundle of a calibrated submanifold is actually isomorphic to an vector bundle intrinsic to the submanifold, as in the abovementioned case of complex hypersurfaces of complex manifolds with trivial 
canonical bundle. In the special Lagrangian case, the normal bundle is isomorphic to the tangent bundle (or cotangent bundle via the metric), while, in the coassociative case, the normal bundle is isomorphic to the bundle of anti-self dual two-forms. In these geometries, the existence of calibrated deformations of a calibrated submanifold will reduce to topological questions of the submanifold itself. It will follow that if the calibrated submanifold is topologically simple (e.g. a sphere), then the submanifold will be rigid as a calibrated submanifold. These results, of course, will be explained and made precise in the appropriate sections.

Layout. The following is a brief summary of the layout of this paper. In the second section, we derive a simple expression for the second variation formula for arbitrary calibrated submanifolds that will be applied to specific calibrated submanifolds in the proceeding sections. In the third section, we study the deformation theory of special Lagrangian submanifolds. In the fourth, we prove results for coassociative submanifolds analogous to those for special Lagrangian submanifolds. In sections five and six, we turn to the other two calibrated geometries given in [HL]: associative three dimensional submanifolds of a seven manifold with holonomy $G_{2}$, and four dimensional Cayley submanifolds of eight dimensional manifolds with holonomy contained in Spin (7). For the calibrated submanifolds of these last two geometries, the normal bundles are not intrinsic. Rather, the normal bundles are actually the spin bundles twisted by extrinsic vector bundles. As a consequence, in these latter cases, the results are not as strong as in the first two cases. Finally, in the last section, we pose questions, and make conjectures.

Remarks on Submanifolds. In this work, a submanifold of $M$ will be an immersion $f: X \rightarrow M$. Two immersions will be considered equivalent if they differ by a diffeomorphism of $X$. The normal bundle will then be a smooth vector bundle on $X$. However, we occasionally lapse into thinking the submanifold $X$ as contained in the ambient manifold, and speak of restriction of forms, metrics, etc., rather than pull-back of these quantities.

In this paper, it will always be assumed that $X$ is compact and orientable. (We do not assume that the ambient manifold $M$ is compact.) The compactness of $X$ has the following consequences. Firstly, Hodge theory applies to $X$. In particular, the Hodge-de Rham theorem identifies the space of harmonic forms on $X$ with closed and co-closed forms and in turn, with the singular cohomology spaces (which are topological invariants). Secondly, a 
deformation of submanifolds, $f_{t}: \tilde{X} \rightarrow X_{t} \subset M$ may be assumed to be a normal deformation, i.e., $\frac{\partial}{\partial t} f_{t}(p)$ is normal to $X_{t}$ for $p \in X_{t}$ fixed. This is because, if $\tilde{X}$ is compact, then one can reparametrize using a time dependent diffeomorphism of $\tilde{X}$. Thirdly, there is a tubular neighborhood of $X$ in $M$ that is identified via the normal exponential map to a neighborhood $N_{\epsilon}(X)=\{V \in N(X) \mid\|V\|<\epsilon\}$ of the zero section in $N(X)$. We can thereby identify nearby submanifolds (in the $C^{1}$ topology) with small normal vector fields. By pulling back various structures of the ambient manifold $M$ (calibrating forms, metrics, complex structures, etc.) to this neighborhood of the zero section in $N(X)$, we may assume that the submanifold is embedded.

Acknowledgements. The author would like to express his gratitude for conversations with Robert Bryant, Reese Harvey, and Lucas Hsu.

\section{The Second Variation of Calibrated Submanifolds.}

In this section we derive a rather remarkable formula for the second variation of a calibrated submanifold. In the usual formulation, the expression for the second variation involves the Riemann curvature of the ambient manifold together with the second fundamental form of the immersion, [Ch1]. In contrast, the formula derived here only involves terms from the restriction of the calibrating form and its covariant derivatives to the submanifold. This section concludes with an example computing the second variation of complex submanifolds of a Kähler manifold.

Let $M^{n}$ be a Riemannian manifold with a calibration $\vartheta \in \Omega^{p}(M)$. Let $f: X^{p} \rightarrow M^{n}$ be a calibrated submanifold, i.e., $f^{*}(\vartheta)=d v o l_{X}$, and let $f_{t}=$ $F: X \times I \rightarrow M$ be a one parameter family of immersions (not necessarily calibrated) with $f_{0}=f$. Since $X$ is assumed compact we may reparametrize so that the family $f_{t}=F$ is orthogonal, i.e., we assume the variation vector field $V=F_{*}\left(\frac{\partial}{\partial t}\right)=\frac{\partial}{\partial t} f_{t}$ is perpendicular to $T(X)$.

Consider now $\vartheta$, not as a differential form, but rather a section of the vector bundle $\Lambda^{p}\left(T^{*} M\right)$. Then one may view $f^{*}(\vartheta)$ as a section of $\Lambda^{p}\left(f^{*}\left(T^{*} M\right)\right)=\Lambda^{p}\left(T^{*} X \oplus N^{*}(X)\right)$. We have the standard decomposition

$$
\Lambda^{p}\left(T^{*} X \oplus N^{*} X\right)=\bigoplus_{k=0}^{\min p, n-p} \Lambda^{p-k}\left(T^{*} X\right) \otimes \Lambda^{k}\left(N^{*} X\right) .
$$

Let $\vartheta_{0}, \vartheta_{1}, \ldots$ be the components of $f^{*}(\vartheta)$ with respect to this decomposition. The zeroth component $\vartheta_{0}$, a section of $\Lambda^{p}\left(T^{*} X\right)$, is just the pullback 
of $\vartheta$ as a differential form. It follows from the "first cousin principle" (see Proposition 2.5 below) that the component $\vartheta_{1}$ will always vanish. The second component $\vartheta_{2}$ plays an important role in what follows.

The component $\vartheta_{2}$ is a section of $\Lambda^{p-2}\left(T^{*} X\right) \otimes \Lambda^{2}\left(N^{*} X\right)$ which by the Hodge star operator and the metric $\sharp$ operator on the first factor is isomorphic to $\Lambda^{2}(T X) \otimes \Lambda^{2}\left(N^{*} X\right)$. Now because of the decomposition

$$
S^{2}\left(T X \otimes N^{*} X\right)=S^{2}(T X) \otimes S^{2}\left(N^{*} X\right) \oplus \Lambda^{2}(T X) \otimes \Lambda^{2}\left(N^{*} X\right),
$$

we see that we may view $\vartheta_{2}$ as a section of $S^{2}\left(T X \otimes N^{*} X\right)$ which is used in the following definition:

Definition 2.1. Let $V \in \Gamma(N X)$ a normal vector field to our calibrated submanifold, and $\nabla V \in \Gamma\left(N X \otimes T^{*} X\right)$ be its covariant derivative. We define $\mathcal{Q}_{\vartheta}(\nabla V)$ to be the complete contraction of $\nabla V \circ \nabla V$ with $\vartheta_{2}$ viewed as a section of $S^{2}\left(T X \otimes N^{*} X\right)$. (See also (2.23), below.)

Consider the pull-back of the covariant derivative $\nabla \vartheta$ as a section of

$$
\begin{aligned}
\Lambda^{p}\left(f^{*}\left(T^{*} M\right)\right) \otimes f^{*}\left(T^{*} M\right)=\Lambda^{p}\left(T^{*} X \oplus N^{*} X\right) \otimes\left(T^{*} X \oplus N^{*} X\right) \\
\quad=\bigoplus_{k=0}^{\min p, n-p} \Lambda^{p-k}\left(T^{*} X\right) \otimes \Lambda^{k}\left(N^{*} X\right) \otimes\left(T^{*} X \oplus N^{*} X\right)
\end{aligned}
$$

Let $(\nabla \vartheta)_{1}$ be the piece of $f^{*}(\nabla \vartheta)$ in the component $\Lambda^{p-1}\left(T^{*} X\right) \otimes N^{*} \otimes N^{*}$ in the above decomposition. Via the musical and Hodge isomorphisms, we may view $(\nabla \vartheta)_{1}$ as taking values in $T X \otimes N^{*} \otimes N^{*}$.

Definition 2.2. For a normal vector field $V \in \Gamma(N X)$ with $\nabla V \in$ $\Gamma\left(N X \otimes T^{*} X\right)$ its covariant derivative, we let $\mathcal{B}_{\nabla \vartheta}(V, \nabla V)$ denote the complete contraction of $V \circ \nabla V$ with $(\nabla \vartheta)_{1}$. (See also (2.24).)

We need another quadratic form. One pulls back the second covariant derivative $\nabla^{2} \vartheta$ to $X$ via $f$ and obtain a section of the bundle

$$
\begin{aligned}
& \Lambda^{p}\left(f^{*}\left(T^{*} M\right)\right) \otimes f^{*}\left(T^{*} M \otimes T^{*} M\right) \\
& \quad=\Lambda^{p}\left(T^{*} X \oplus N^{*} X\right) \otimes\left(T^{*} X \oplus N^{*} X\right) \otimes\left(T^{*} X \oplus N^{*} X\right) .
\end{aligned}
$$

We denote by $\left(\nabla^{2} \vartheta\right)_{0}$ the piece of $f^{*}\left(\nabla^{2} \vartheta\right)$ taking values in the component $\Lambda^{p}\left(T^{*} X\right) \otimes S^{2}\left(N^{*}\right)$. Via the Hodge star isomorphism, we realize $\left(\nabla^{2} \vartheta\right)_{0}$ as a section of $S^{2}\left(N^{*}\right)$. 
Definition 2.3. For a normal vector field $V \in \Gamma(N X)$, we let $\mathcal{Q}_{\nabla^{2} \vartheta}(V)$ denote the complete contraction of $V \circ V$ with $\left(\nabla^{2} \vartheta\right)_{0}$. (See also (2.22).)

Theorem 2.4. The second variation of volume of a calibrated submanifold $X^{p}$ with variation vector field $V$ is given by

$\left.\frac{d^{2}}{d t^{2}} \operatorname{vol}\left(X_{t}\right)\right|_{t=0}=\int_{X}\|\nabla V\|^{2}-\left(\mathcal{Q}_{\vartheta}(\nabla V)+\mathcal{B}_{\nabla \vartheta}(V, \nabla V)+\mathcal{Q}_{\nabla^{2} \vartheta}(V)\right) d v \operatorname{vol}_{X}$.

Remark. If the calibration is parallel $(\nabla \vartheta=0)$ which is the only type of calibration considered here, then the $\mathcal{B}_{\nabla \vartheta}$ and $\mathcal{Q}_{\nabla^{2} \vartheta}$ terms do not appear. It is also interesting to note that in the case $\nabla \vartheta=0$ the second variation only depends on $\nabla V$.

Proof ( Preliminaries ). Since $\vartheta(t)$ and $d v o l(t)$ are both top dimensional forms on $X$ we may write $\vartheta(t)$ as $\lambda(t) d \operatorname{vol}(t)$, for some function $\lambda(t)$. By our assumption that the original submanifold is calibrated we must have $\lambda(0)=1$. Now we have

$$
\text { Volume of } \begin{aligned}
X_{0} & =\int_{X_{0}} \vartheta \\
& =\int_{X_{t}} \vartheta \quad \text { by Stoke's theorem. } \\
& =\int_{X} \vartheta(t) \\
& =\int_{X} \lambda(t) d \operatorname{vol}(t)
\end{aligned}
$$

In particular, $\int_{X} \lambda(t) d \operatorname{vol}(t)$ is constant in $t$. Differentiating in time and evaluating at $t=0$, we obtain

$$
0=\int_{X} \frac{\partial \lambda}{\partial t}(0) d \operatorname{vol}(0)+\left.\int_{X} \frac{\partial}{\partial t} d \operatorname{vol}(t)\right|_{t=0} .
$$

Differentiating again and evaluating at $t=0$, we obtain

$$
0=\int_{X} \frac{\partial^{2} \lambda}{\partial t^{2}}(0) d \operatorname{vol}(0)+\left.\int_{X} \frac{\partial \lambda}{\partial t}(0) \frac{\partial}{\partial t} d \operatorname{vol}(t)\right|_{t=0}+\left.\int_{X} \frac{\partial^{2}}{\partial t^{2}} d \operatorname{vol}(t)\right|_{t=0},
$$

or

$$
\frac{d^{2}}{d t^{2}} \int_{X} d \operatorname{vol}(t)=-\int_{X} \frac{\partial^{2} \lambda}{\partial t^{2}}(0) d \operatorname{vol}(0)-\left.\int_{X} \frac{\partial \lambda}{\partial t}(0) \frac{\partial}{\partial t} d \operatorname{vol}(t)\right|_{t=0}
$$


The theorem will then follow by showing

$$
\begin{aligned}
\frac{\partial \lambda}{\partial t}(0) & =0 \\
\frac{\partial^{2} \lambda}{\partial t^{2}}(0) & =-\|\nabla V\|^{2}+\left(\mathcal{Q}_{\vartheta}(\nabla V)+\mathcal{B}_{\nabla \vartheta}(V, \nabla V)+\mathcal{Q}_{\nabla^{2} \vartheta}(V)\right) .
\end{aligned}
$$

Proof of (2.8). We prove the theorem by verifying (2.8), using the moving frame, so we first recall various notions from the theory.

Define the orthonormal coframe bundle of $(M, g)$ as

$$
\mathcal{F}(M)=\left\{u:\left(T_{x} M, g_{x}\right) \rightarrow\left(\mathbb{R}^{n}, g_{0}\right) \mid u \text { a linear isometry }\right\} .
$$

Let $\omega$ be the canonical $\mathbb{R}^{n}$-valued 1-form on $\mathcal{F}(M)$ defined as by $\omega(V)=$ $u\left(\pi_{*}(V)\right)$ where $\pi$ is the projection of $\mathcal{F}(M)$ to $M$, and $V \in T_{u} \mathcal{F}(M)$. Let $\varphi$ denote the $\mathfrak{s o}(n)$ valued Levi-Civita connection form satisfying $d \omega=-\varphi \wedge \omega$. Fix the index range

$$
1 \leq A, B, C, D \leq n \text {. }
$$

Choosing an orthonormal basis $e_{A}$ of $\mathbb{R}^{n}$, we write $\omega=\omega^{A} e_{A}, \varphi=\varphi_{B}^{A} e_{A} \otimes$ $\left(e_{B}\right)^{*}$ so that $d \omega^{A}=-\varphi_{B}^{A} \wedge \omega^{B}$. The Riemannian curvature of the ambient manifold is defined by $d \varphi_{B}^{A}+\varphi_{C}^{A} \wedge \varphi_{B}^{C}=(1 / 2) R_{B C D}^{A} \omega^{C} \wedge \omega^{D}$.

Let $\vartheta$ be a calibration on $M$. We pull back $\vartheta$ to the coframe bundle $\mathcal{F}(M)$ where we have $\vartheta=\lambda_{A_{1} \cdots A_{p}} \omega^{A_{1}} \wedge \cdots \wedge \omega^{A_{p}}$. We define the first covariant derivative of $\vartheta$ by

$$
d \lambda_{A_{1} \cdots A_{p}}-\lambda_{B A_{2} \cdots A_{p}} \varphi_{A_{1}}^{B}-\cdots-\lambda_{A_{1} \cdots A_{p-1} B} \varphi_{A_{p}}^{B}=\lambda_{A_{1} \cdots A_{p}, B} \omega^{B}
$$

where $\lambda_{A_{1} \ldots A_{p}, B}$ represent the components of $\nabla \vartheta$. We will also need $\nabla^{2} \vartheta$; whose components $\lambda_{A_{1} \cdots A_{p}, B C}$ are defined by

$$
d \lambda_{A_{1} \cdots A_{p}}-\lambda_{C A_{2} \cdots A_{p}, B} \varphi_{A_{1}}^{C}-\cdots-\lambda_{A_{1} \cdots A_{p}, C} \varphi_{B}^{C}=\lambda_{A_{1} \cdots A_{p}, B C} \omega^{C},
$$

and are subject to the Bianchi-type identity:

$$
\lambda_{D A_{2} \cdots A_{p}} R_{A_{1} B C}^{D}+\cdots+\lambda_{A_{1} \cdots A_{p-1} D} R_{A_{p} B C}^{D}=\lambda_{A_{1} \cdots A_{p}, B C}-\lambda_{A_{1} \cdots A_{p}, C B}
$$

(which follows from $d^{2} \vartheta=0$.)

Suppose now one has a one-parameter family of immersions $f_{t}=F$ : $X^{p} \times I \rightarrow M$ such that $f=f_{0}: X \rightarrow M$ is calibrated by $\vartheta$. We may 
reparametrize so that the family of immersions is normal and then adapt frames as usual, by restricting to the subbundle $\mathcal{F}^{(1)}$ of $\mathcal{F}^{(0)}=F^{*}(\mathcal{F}(M))$ such that $\left.\omega^{a}=V^{a} d t, \frac{\partial}{\partial t}\right\lrcorner \omega^{i}=0$ where we fix the index ranges $1 \leq i, j, k \leq p$ and $p+1 \leq a, b, c \leq n$.

The structure equations for the immersion may be written

$$
\left.d\left(\begin{array}{c}
\omega^{i} \\
\omega^{a}
\end{array}\right)=-\left(\begin{array}{ll}
\varphi_{j}^{i} & \varphi_{b}^{i} \\
\varphi_{j}^{a} & \varphi_{b}^{a}
\end{array}\right) \wedge\left(\begin{array}{c}
\omega^{j} \\
\omega^{b}
\end{array}\right), \quad \omega^{a}=V^{a} d t, \frac{\partial}{\partial t}\right\lrcorner \omega^{i}=0
$$

Differentiating $\omega^{a}=V^{a} d t$ we find

$$
\varphi_{i}^{a}=-\varphi_{a}^{i}=h_{i j}^{a} \omega^{j}+V_{i}^{a} d t, \quad \text { where } \quad h_{i j}^{a}=h_{j i}^{a},
$$

where we have set

$$
d V^{a}+\varphi_{b}^{a} V^{b}=V_{i}^{a} \omega^{i}+\dot{V}^{a} d t .
$$

Restricting $\vartheta$ to the adapted frame bundle $\mathcal{F}^{(1)}$, we obtain

$$
\vartheta=p !(\lambda_{1 \cdots p} \omega^{1} \wedge \cdots \wedge \omega^{p}+\lambda_{1 \cdots a} i_{\cdots p} \omega^{1} \wedge \cdots \wedge \overbrace{\omega^{a}}^{i} \wedge \cdots \wedge \omega^{p}) .
$$

Observation. For fixed times (whence $\omega^{a}=0$ ), we see that

$$
\vartheta=p ! \lambda_{1 \cdots p} \omega^{1} \wedge \cdots \wedge \omega^{p},
$$

and hence the quantity $\lambda=\lambda(t)$ introduced above is $p ! \lambda_{1 \ldots p}$. Hence to verify (2.8), we need the time derivatives of $\lambda_{1 \ldots p}$.

By (2.9)

$$
d \lambda_{1 \cdots p}=\lambda_{1 \cdots A} \stackrel{i}{i \cdots p} \varphi_{i}^{A}+\lambda_{1 \cdots p, A} \omega^{A}
$$

and so with the aid of formula (2.12) and since $\omega^{a}=V^{a} d t$, we have

$$
\begin{aligned}
\frac{\partial}{\partial t} \lambda_{1 \cdots p} & \left.=\frac{\partial}{\partial t}\right\lrcorner d \lambda_{1 \cdots p} \\
& =\lambda_{1 \cdots a} \cdots p V_{i}^{a}+\lambda_{1 \cdots p, a} V^{a} .
\end{aligned}
$$

\section{Proposition 2.5 (Generalized First Cousin Principle).}

$$
\left.\lambda_{1 \cdots a} \cdots p\right|_{t=0}=0 \quad \text { and }\left.\quad \lambda_{1 \cdots p, a}\right|_{t=0}=0 .
$$


Proof. Associated to the calibration $\vartheta$ there is a function $\ell_{\vartheta}: \tilde{G}(p, T(M)) \rightarrow$ $\mathbb{R}$ where $\tilde{G}(p, T(M))$ is the bundle of oriented $p$-planes $\xi$ of the tangent spaces of $M$ where if $v_{1}, \ldots, v_{p}$ is an oriented orthonormal basis for $\xi$, then $\ell_{\vartheta}(\xi)$ is $\vartheta\left(v_{1} \wedge \cdots \wedge v_{p}\right)$. Recalling (2.9), there is a projection $\pi: \mathcal{F}(M) \rightarrow$ $\tilde{G}(p, T(M))$ defined by $\pi(u)=u^{-1}\left(e_{1} \wedge \cdots \wedge e_{p}\right)$. The forms $\omega^{i}, \omega^{a}$, and $\varphi_{i}^{a}$ are then semi-basic for the projection to $\tilde{G}(p, T(M))$. The function $\ell_{\vartheta}$ : $\tilde{G}(p, T(M)) \rightarrow \mathbb{R}$ pulled back to $\mathcal{F}(M)$ is just $\lambda_{1 \cdots p}$. By definition of a calibration, $\ell_{\vartheta}$ takes values in $[-1,1]$ where a $p$-plane $\xi$ is calibrated if $\ell_{\vartheta}(\xi)=$ 1 . Such a $p$-plane is obviously a critical point for the function $\ell_{\vartheta}$. Since by (2.17),

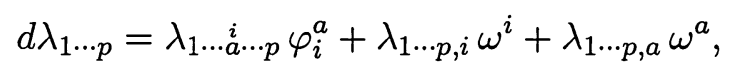

and so at a critical point of $\ell_{\vartheta}$, i.e., a calibrated $p$-plane the components of the exterior derivative $\lambda_{1 \cdots a} \underset{i}{i}, \lambda_{1 \cdots p, i}$, and $\lambda_{1 \cdots p, a}$ vanish. Finally, given an immersion $f: X \rightarrow M$ of an oriented manifold $X$, there is a canonical lift $\tilde{f}: X \rightarrow \tilde{G}(p, T(M))$, and if $X$ is calibrated then all the points of $X$ are critical points of $\ell_{\vartheta}$ and $\lambda_{1 \cdots a} \ldots p, \lambda_{1 \cdots p, i}$, and $\lambda_{1 \cdots p, a}$ vanish.

Remark. The original first cousin principle dealt with constant coefficient calibrations in flat space, [H] and [HL, page 78]. In particular, $\left.\lambda_{1 \cdots p, a}\right|_{t=0}=0$ was satisfied trivially. Also, combining equations (2.5) and (2.16) and the previous proposition; one obtains an infinitesimal proof that the calibrated submanifolds are minimal.

We turn now to the calculation of the second derivative of $\lambda=p ! \lambda_{1 \ldots p}$, differentiating (2.16). We set

$$
d V_{i}^{a}+\varphi_{b}^{a} V_{i}^{b}-\varphi_{i}^{j} V_{j}^{a}=V_{i j}^{a} \omega^{j}+\dot{V}_{i}^{a} d t
$$


Differentiating the first term on the right hand side of (2.16), we find

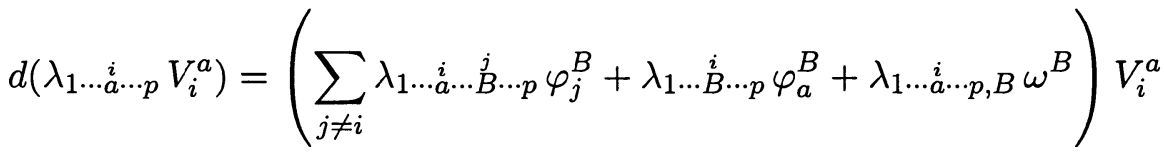

$$
\begin{aligned}
& +\lambda_{1 \cdots a} \stackrel{i}{a} \cdots p\left(\varphi_{i}^{j} V_{j}^{a}-\varphi_{b}^{a} V_{i}^{b}+V_{i j}^{a} \omega^{j}+\dot{V}_{i}^{a} d t\right)
\end{aligned}
$$

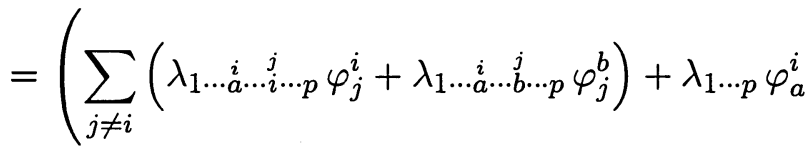

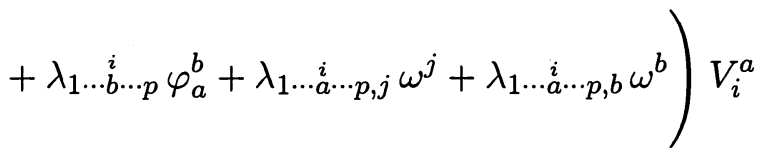

$$
\begin{aligned}
& +\lambda_{1 \cdots a \cdots p}\left(\varphi_{i}^{j} V_{j}^{a}-\varphi_{b}^{a} V_{i}^{b}+V_{i j}^{a} \omega^{j}+\dot{V}_{i}^{a} d t\right) .
\end{aligned}
$$

So with (2.12) and our normalization $\left.\omega^{a}=V^{a} d t, \frac{\partial}{\partial t}\right\lrcorner \omega^{i}=0$, we find

$$
\begin{aligned}
& \left.\frac{\partial}{\partial t}\left(\lambda_{1 \ldots a}^{i} \ldots p V_{i}^{a}\right)=\frac{\partial}{\partial t}\right\lrcorner d\left(\lambda_{1 \ldots a} \ldots p V_{i}^{a}\right)
\end{aligned}
$$

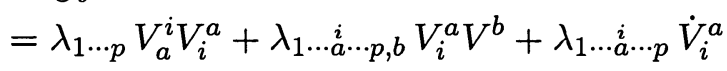

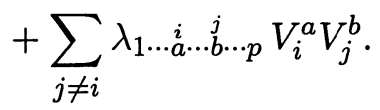

(where $V_{a}^{i}=-V_{i}^{a}$.)

Next, differentiating the second term of the right hand side of (2.16), we compute

$$
\begin{aligned}
& d\left(\lambda_{1 \cdots p, a} V^{a}\right)=\left(\lambda_{1 \cdots B} \stackrel{i}{\cdots p, a} \varphi_{i}^{B}+\lambda_{1 \cdots p, B} \varphi_{a}^{B}+\lambda_{1 \cdots p, a B} \omega^{B}\right) V^{a} \\
& +\lambda_{1 \cdots p, a}\left(-\varphi_{b}^{a} V^{b}+V_{i}^{a} \omega^{i}+\dot{V}^{a} d t\right) \\
& =\left(\lambda_{1 \cdots b \cdots p, a}^{i} \varphi_{i}^{b}+\lambda_{1 \cdots p, i} \varphi_{a}^{i}+\lambda_{1 \cdots p, b} \varphi_{b}^{i}\right. \\
& \left.+\lambda_{1 \cdots p, a i} \omega^{i}+\lambda_{1 \cdots p, a b} \omega^{b}\right) V^{a} \\
& +\lambda_{1 \cdots p, a}\left(-\varphi_{b}^{a} V^{b}+V_{i}^{a} \omega^{i}+\dot{V}^{a} d t\right),
\end{aligned}
$$

so, again using (2.12) and $\left.\omega^{a}=V^{a} d t, \frac{\partial}{\partial t}\right\lrcorner \omega^{i}=0$, we find

$$
\begin{aligned}
\frac{\partial}{\partial t}\left(\lambda_{1 \cdots p, a} V^{a}\right)= & \left.\frac{\partial}{\partial t}\right\lrcorner d\left(\lambda_{1 \cdots p, a} V^{a}\right) \\
= & \lambda_{1 \cdots b \cdots p, a} V_{i}^{b} V^{a}+\lambda_{1 \cdots p, i} V_{a}^{i} V^{a} \\
& +\lambda_{1 \cdots p, a b} V^{a} V^{b}+\lambda_{1 \cdots p, a} V^{a} .
\end{aligned}
$$


Lemma 2.6. At $t=0, \lambda_{1 \cdots p, a b}=\lambda_{1 \cdots p, b a}$.

Proof. This follows from the Bianchi identity (2.11). In particular, from (2.11) we have

$$
\begin{aligned}
\lambda_{1 \cdots p, b c}-\lambda_{1 \cdots p, c b} & =\lambda_{D 2 \cdots p} R_{1 b c}^{D}+\cdots+\lambda_{1 \cdots(p-1) D} R_{p b c}^{D} \\
& =\lambda_{a 2 \cdots p} R_{1 b c}^{a}+\cdots+\lambda_{1 \cdots(p-1) a} R_{p b c}^{a}
\end{aligned}
$$

and from the proposition, at $t=0, \lambda_{a 2 \cdots p}=\cdots=\lambda_{1 \cdots(p-1) a}=0$, and the lemma follows.

By the preceeding lemma, we need not worry about symmetrizing in the following equation which defines $\mathcal{Q}_{\nabla^{2} \vartheta}$ :

$$
\mathcal{Q}_{\nabla^{2} \vartheta}(V)=p ! \lambda_{1 \cdots p, a b} V^{a} V^{b} .
$$

Also set

$$
\mathcal{Q}_{\vartheta}(\nabla V)=p ! \sum_{j \neq i} \lambda_{1 \cdots j \cdots b} \underset{i j}{j} \ldots p V_{i}^{a} V_{j}^{b}
$$

and

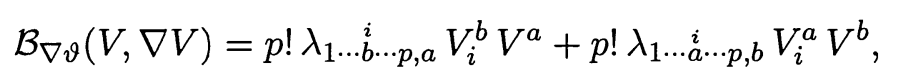

and note

$$
\lambda_{1 \cdots p} V_{a}^{i} V_{i}^{a}=-\frac{1}{p !} V_{i}^{a} V_{i}^{a}=-\frac{1}{p !}\|\nabla V\|^{2}
$$

So combining (2.20) and (2.21), and the vanishing of terms implied by Proposition 2.5 , we have

$$
\begin{aligned}
\frac{\partial^{2}}{\partial t^{2}} \lambda & =\frac{\partial^{2}}{\partial t^{2}} p ! \lambda_{1 \cdots p} \\
& =-\|\nabla V\|^{2}+\left(\mathcal{Q}_{\vartheta}(\nabla V)+\mathcal{B}_{\nabla \vartheta}(V, \nabla V)+\mathcal{Q}_{\nabla^{2} \vartheta}(V)\right) .
\end{aligned}
$$

With $\left(2.7^{\prime}\right)$, the theorem is proved.

Example. We conclude this section with one example, that of Simons' result on the second variation of complex submanifolds of Kähler manifolds, specialized to the case of complex curves $\Sigma$ in a complex Kähler surface $M$ 
(complex co-dimension 1.) We leave the general case of higher dimensions and co-dimensions to the reader.

Either by working with a local coframe or by working on the unitary frame bundle of $M$ (see [Ch2]), one may assume a unitary coframe $\omega^{1}+$ $i \omega^{2}, \omega^{3}+i \omega^{4}$, so that the Kähler form is given by $\vartheta=\lambda_{A B} \omega^{A} \wedge \omega^{B}=$ $\omega^{1} \wedge \omega^{2}+\omega^{3} \wedge \omega^{4}$, and $\omega^{3},\left.\omega^{4}\right|_{\Sigma}=0,\left.\vartheta\right|_{\Sigma}=\omega^{1} \wedge \omega^{2}$ is the volume form of $\Sigma$. In particular, $\lambda_{34}=1 / 2$, and so one computes (2.23) that

$$
\begin{aligned}
\mathcal{Q}_{\vartheta}(\nabla V) & =2 \lambda_{a b} V_{1}^{a} V_{2}^{b}+2 \lambda_{b a} V_{2}^{a} V_{1}^{b} \\
& =4 \lambda_{a b} V_{1}^{a} V_{2}^{b}=2 V_{1}^{3} V_{2}^{4}-2 V_{1}^{4} V_{2}^{3} .
\end{aligned}
$$

Hence

$$
\begin{aligned}
\|\nabla \vartheta\|^{2}-\mathcal{Q}_{\vartheta}(\nabla V)= & \left(V_{1}^{3}\right)^{2}+\left(V_{2}^{3}\right)^{2}+\left(V_{1}^{4}\right)^{2} \\
& +\left(V_{2}^{4}\right)^{2}-2\left(V_{1}^{3} V_{2}^{4}-V_{1}^{4} V_{2}^{3}\right) \\
= & \left(V_{1}^{3}-V_{2}^{4}\right)^{2}+\left(V_{2}^{3}+V_{1}^{4}\right)^{2} .
\end{aligned}
$$

(The Kähler form is parallel, so the other terms in the integrand of (2.5) do not appear.) If we set $V=V^{3}+i V^{4}$, then

$$
\begin{aligned}
d V= & \frac{1}{2}\left(\left(V_{1}^{3}+V_{2}^{4}\right)+i\left(V_{1}^{4}-V_{2}^{3}\right)\right) \omega \\
& +\frac{1}{2}\left(\left(V_{1}^{3}-V_{2}^{4}\right)+i\left(V_{2}^{3}+V_{1}^{4}\right)\right) \bar{\omega}
\end{aligned}
$$

where $\omega=\omega^{1}+i \omega^{2}$. Therefore

$$
\bar{\partial} V=\frac{1}{2}\left(\left(V_{1}^{3}-V_{2}^{4}\right)+i\left(V_{2}^{3}+V_{1}^{4}\right)\right) \bar{\omega}
$$

and so

$$
\|\bar{\partial} V\|^{2}=\frac{1}{2}\left(\left(V_{1}^{3}-V_{2}^{4}\right)^{2}+\left(V_{2}^{3}+V_{1}^{4}\right)^{2}\right)=|\nabla \vartheta|^{2}-\mathcal{Q}_{\vartheta}(\nabla V) .
$$

Combining (2.29) and our formula (2.5) for the second variation, we recover Simons' result:

$$
\left.\frac{d^{2}}{d t^{2}} \operatorname{vol}\left(X_{t}\right)\right|_{t=0}=2 \int_{X}\|\bar{\partial} V\|^{2} d \operatorname{vol}_{X}
$$

\section{Deformations of Special Lagrangian Submanifolds.}

Since special Lagrangian submanifolds lie in $S U(n)$ manifolds, we first develop the structure equations for the ambient $S U(n)$ manifold $M^{2 n}$. We 
begin with constructions in flat space which will then be repeated on the tangent spaces of $M^{2 n}$. In this section, we take the index range $1 \leq i, j, k, l \leq n$, and use the notation that a primed index, say $i^{\prime}$, denotes the index with value $i+n$.

On $\mathbb{C}^{n}$, take standard coordinates

$$
z^{1}=x^{1}+i x^{n+1}, \ldots, z^{n}=x^{n}+i x^{2 n}
$$

and let $h_{0}=\sum d z^{i} \otimes d \bar{z}^{i}$ be the standard hermitian metric on $\mathbb{C}^{n}$. Finally, we let $\zeta_{0}=d z^{1} \wedge \cdots \wedge d z^{n}$ be the complex determinant form. Viewing $\mathbb{C}^{n}$ as $\mathbb{R}^{2 n}$, we have on $\mathbb{R}^{2 n}$ the following structures:

(1) A complex structure $J_{0}$, which is simply multiplication by $\sqrt{-1}$, viewed as a real map. In particular, $J_{0}\left(\frac{\partial}{\partial x^{i}}\right)=\frac{\partial}{\partial x^{i^{i}}}$ and $J_{0}\left(\frac{\partial}{\partial x^{i^{i}}}\right)=-\frac{\partial}{\partial x^{i}}$.

(2) A metric $g_{0}=\operatorname{Re}\left(h_{0}\right)=\left(d x^{1}\right)^{2}+\left(d x^{n+1}\right)^{2}+\cdots+\left(d x^{n}\right)^{2}+\left(d x^{2 n}\right)^{2}$.

(3) A symplectic form, the Kähler form,

$$
\kappa_{0}=\operatorname{Im}\left(h_{0}\right)=d x^{1} \wedge d x^{n+1}+\cdots+d x^{n} \wedge d x^{2 n} .
$$

(which is compatible with the metric and complex structure in the sense that $\kappa_{0}(\cdot, \cdot)=g_{0}\left(\cdot, J_{0} \cdot\right)$.)

(4) Two (real) $n$-forms $\alpha_{0}$ and $\beta_{0}$, the real and imaginary parts of $\zeta$, respectively.

Now, a real $n$-plane in $\mathbb{C}^{n}$, is said to be special Lagrangian if the form $\alpha_{0}$ restricts to be the volume form. For example, it is easy to see that $\xi^{n}=\frac{\partial}{\partial x_{1}} \wedge \cdots \wedge \frac{\partial}{\partial x_{n}}$ is special Lagrangian. For such $n$-planes, we have the easily verified

Lemma 3.1. The group $S U(n)$ acts transitively on the set of special Lagrangian n-planes with isotropy $S O(n)$.

Next, we place these structures on the tangent spaces of $M$ : We say that $M$ has an almost $S U(n)$ structure if $M$ carries an almost complex structure $J$, a hermitian metric $h=g+i \kappa$, and a complex volume form $\zeta$ (i.e., a nowhere vanishing complex form of type $(n, 0))$. We call the $S U(n)$ structure integrable if: 1) the almost complex structure, 2) the Kähler form, and 3) the complex volume form are covariant constant with respect to Levi-Civita connection of the Riemannian metric $g$. $J$ being covariant constant implies the manifold $M$ will in fact be a Kähler manifold, in particular, a complex 
manifold. Also, the form $\zeta$ will be closed and hence holomorphic and $\zeta$ will trivialize the canonical bundle. We will simply say that $M$ is an $S U(n)$ manifold or that $M$ has an $S U(n)$ structure if these conditions are satisfied. Such manifolds are also called special Kähler manifolds or Calabi-Yau manifolds. Their existence and abundance was demonstrated by Yau in his proof of the Calabi conjecture, see [Y]. For example, any smooth hypersurface of degree $(n+1)$ in $\mathbb{C P}^{n}$ has an $S U(n)$ stucture.

Next we develop the $S U(n)$ coframe bundle $\mathcal{F}_{S U(n)}(M)=\mathcal{F}(M)$ for $M$ (compare [Br2]). For a point $x \in M$, let $\mathcal{F}_{x}(M)=\left\{u: T_{x}(M) \rightarrow \mathbb{C}^{n}\right\}$ where we require that $u$ is complex linear, $u$ is an isometry taking $h$ to $h_{0}$, and that $u$ takes $\zeta$ to $\zeta_{0}$. We write the components of the canonical $\mathbb{C}^{n}$-valued form on $\mathcal{F}$ as $\omega_{i}+i \eta_{i}$. Then we have:

$$
\begin{aligned}
& \kappa=\omega^{1} \wedge \omega^{n+1}+\cdots+\omega^{n} \wedge \omega^{2 n} \\
& \zeta=\left(\omega^{1}+i \omega^{n+1}\right) \wedge \cdots \wedge\left(\omega^{n}+i \omega^{2 n}\right)
\end{aligned}
$$

The structure equations then take the form:

$$
d\left(\begin{array}{c}
\omega^{i} \\
\omega^{i^{\prime}}
\end{array}\right)=\left(\begin{array}{rr}
\rho_{j}^{i} & \sigma_{j}^{i} \\
-\sigma_{j}^{i} & \rho_{j}^{i}
\end{array}\right) \wedge\left(\begin{array}{c}
\omega^{j} \\
\omega^{j^{\prime}}
\end{array}\right)
$$

where $\rho_{j}^{i}=-\rho_{i}^{j}$ and $\sigma_{j}^{i}=\sigma_{i}^{j}$ and $\operatorname{trace}(\sigma)=0$. The integrability of the $S U(n)$ structure guarantees the absence of torsion. In fact, the integrability of the $S U(n)$ structure is equivalent the existence of a torsion free connection on $\mathcal{F}_{S U(n)}(M)$, see $[\mathrm{Br} 1]$.

A real $n$-dimensional submanifold $X$ of $M$ is said to be special Lagrangian if each tangent space is special Lagrangian. Thus, the form $\alpha$ restricts to $X$ to be the volume form. From [HL], we take the following alternative characterization of special Lagrangian submanifolds:

Proposition 3.2. A n-dimensional submanifold $X$ is special Lagrangian if and only if the forms $\kappa$ and $\beta$ restrict to $X$ to zero.

Proof. See [HL], Corollary III.1.11.

Corollary 3.3. The normal bundle $N(X)$ is isomorphic to the tangent bundle $T(X)$.

Proof. Since $\kappa(\cdot, \cdot)=g(\cdot, J \cdot)$, then $\left.\kappa\right|_{X}=0$ is equivalent to $J$ maps tangent vectors of $X$ to normal vectors of $X$. It follows that $J$ induces an isomorphism of $T(X)$ with $N(X)$. 
Remark 3.4. Using the induced metric isomorphism $b: T(X) \rightarrow T^{*}(X)$, we further obtain an isomorphism of $N(X)$ with $T^{*}(X)$. Thus, we have an identification of normal vector fields to a special Lagrangian submanifold with differential 1-forms on the submanifold. Explicitly, the normal vector field $V=V^{i^{\prime}} \frac{\partial}{\partial \omega^{i^{\prime}}}$ is identified with the 1-form $v=V_{i} \omega^{i}$ with $V^{i^{\prime}}=V_{i}$. This identification will be important in what follows.

Corollary 3.5. If the ambient submanifold $M$ is a torus, then a special Lagrangian submanifold $X$ must have $T(X) \oplus T(X)$ trivial. In particular, all Pontryagin classes of $X$ must vanish.

Proof. $\left.T(X) \oplus T(X) \cong T(X) \oplus N(X) \cong T(M)\right|_{X}$ and $T(M)$ is trivial by hypothesis.

This corollary is vacuous for $X$ of dimension two or three but is nontrivial in higher dimensions. In particular, the signature of a four dimensional special Lagrangian submanifold in a complex four-torus must vanish. This follows from the signature theorem in dimension four.

With these preliminaries, we turn now to the deformation theory of special Lagrangian submanifolds. We have the following

Theorem 3.6. A normal vector field $V$ to a compact special Lagrangian submanifold $X^{n}$ is the deformation vector field to a normal deformation through special Lagrangian submanifolds if and only if the corresponding 1-form $(J V)^{b}$ is closed and co-closed, i.e., harmonic. Thus, we are asserting: First, the Zariski tangent space at $X^{n}$ to the moduli space of special Lagrangian submanifolds is naturally identified with $\mathcal{H}^{1}\left(X^{n}\right)$, the space of harmonic 1-forms. Second, in contrast to the case of complex submanifolds, there are no obstructions in extending a first order special Lagrangian deformation to an actual special Lagrangian deformation.

Corollary 3.7. A special Lagrangian submanifold $X^{n}$ of a flat $2 n$-dimensional torus equipped with a $S U(n)$ structure will have $\beta^{1}(X)=$ $\operatorname{dim}\left(H^{1}(X, \mathbb{R})\right) \geq n$.

Proof of Corollary 3.7. The $2 n$ Killing vector fields will project to $N(X)$ yielding at least $n$ linearly independent normal vector fields which obviously give rise to special Lagrangian deformations. 
Corollary 3.8. If $X$ is a compact special Lagrangian submanifold with $\beta^{1}(X)=0$, then $X$ is rigid as a special Lagrangian submanifold.

More generally, we have the following

Corollary 3.9. The moduli space $\mathcal{M}$ of special Lagrangian submanifolds near $X$ is a smooth manifold of dimension $\beta^{1}(X)$.

Corollary 3.10. The moduli space $\mathcal{M}$ carries a Riemannian metric $g_{\mathcal{M}}$ defined as follows: Given two tangent vectors $v_{1}, v_{2} \in T_{X}(\mathcal{M})$, identify them with harmonic 1-forms $\theta_{1}$ and $\theta_{2}$ and define $g_{\mathcal{M}}\left(v_{1}, v_{2}\right)$ to be $\int_{X}\left\langle\theta_{1}, \theta_{2}\right\rangle d$ vol $_{X}$. Here $\langle\cdot, \cdot\rangle$ is the pointwise inner product on 1-forms.

Remark 3.11. The moduli space also carries an $n$-form $\Theta_{\mathcal{M}}$ : Given $n$ tangent vectors $v_{1}, \ldots, v_{n} \in T_{X}(\mathcal{M})$, identify them with harmonic 1-forms $\theta_{1}, \ldots, \theta_{n}$ on $X$ and define $\Theta_{\mathcal{M}}\left(v_{1}, \ldots, v_{n}\right)$ to be $\int_{X} \theta_{1} \wedge \cdots \wedge \theta_{n}$.

Proof of Theorem 3.6. We define a non-linear map

$$
F: U \subset \Gamma(N(X)) \rightarrow \Omega^{n}(X) \oplus \Omega^{2}(X)
$$

as follows: For a small normal vector field $V=V_{i} \frac{\partial}{\partial \eta_{i}} \in U$, then

$$
F(V)=\left(\left(\exp _{V}\right)^{*}(\beta),\left(\exp _{V}\right)^{*}(-\kappa)\right)
$$

Here $U$ is an open neighborhood of the zero in $\Gamma(N(X))$ for which $V \in U$ implies that the exponential map $\exp _{V}$ is a diffeomorphism of $X$ onto its image $X_{V}$. Under the identification of small normal vector fields with nearby submanifolds, it is easy to see that $F^{-1}(0,0)$ corresponds to the set of nearby special Lagrangian submanifolds: $F$ is the restriction of $\beta$ and $-\kappa$ to $X_{V}$, then pulled back to $X$ via $\exp _{V}{ }^{*}$. Hence, $F^{-1}(0,0)$ is simply the set of normal vector fields $V$ in $U$ for which $\beta$ and $\kappa$ restrict to $X_{V}$ to be zero, i.e., $X_{V}$ is special Lagrangian by Proposition 3.2.

We now compute the linearization of $F$,

$$
F^{\prime}(0): \Gamma(N(X)) \rightarrow \Omega^{n}(X) \oplus \Omega^{2}(X), \text { where } F^{\prime}(0)(V)=\frac{\partial}{\partial t} F(t V) .
$$

Therefore

$$
\begin{aligned}
F^{\prime}(0)(V) & =\left.\frac{\partial}{\partial t} F(t V)\right|_{t=0} \\
& =\left(\left.\mathcal{L}_{V}(\beta)\right|_{X},-\left.\mathcal{L}_{V}(\kappa)\right|_{X}\right) \\
& \left.\left.\left.=((V\lrcorner d \beta+d(V\lrcorner \beta))\left.\right|_{X},-(V\lrcorner d \kappa+d(V\lrcorner \kappa\right)\right)\left.\right|_{X}\right) \\
& \left.\left.=\left.(d(V\lrcorner \beta)\right|_{X},-d(V\lrcorner \kappa\right)\left.\right|_{X}\right) .
\end{aligned}
$$


Here $\mathcal{L}_{V}$ denotes Lie derivative, and the Cartan formula is used. Actually, one technical point is being glossed over. In order to take the Lie derivative, one must extend the normal vector field of the submanifold, and then show the above expression is independent of extension. Arguments of this sort may be found in $[\mathrm{Gr}]$. Now

$$
\left.-V\lrcorner \kappa=-\left(V^{i^{\prime}} \frac{\partial}{\partial \omega^{i^{\prime}}}\right)\right\lrcorner\left(\omega^{i} \wedge \omega^{i^{\prime}}\right)=V^{i^{\prime}} \omega^{i}=V_{i} \omega^{i}=v
$$

where $v$ is the 1-form corresponding $V$ under the identification of $N(X)$ with $T^{*}(X)$. Also,

$$
\begin{aligned}
(V\lrcorner \beta)\left.\right|_{X} & \left.=\left(V^{i^{\prime}} \frac{\partial}{\partial \omega^{i^{\prime}}}\right)\right\lrcorner\left.\operatorname{Im}\left(\omega^{1}+i \omega^{1^{\prime}}\right) \wedge \cdots \wedge\left(\omega^{n}+i \omega^{n^{\prime}}\right)\right|_{X} \\
& =V_{1} * \omega^{1}+\cdots V_{n} * \omega^{n} \\
& =* v
\end{aligned}
$$

Substituting (3.6), (3.7) into (3.5), we see that $F^{\prime}(0)(V)=\left(* d^{*} v, d v\right)$. Hence, $F^{\prime}(0)$ as a map $F^{\prime}(0): \Omega^{1}(X) \rightarrow \Omega^{n}(X) \oplus \Omega^{2}(X)$ is just $-* d^{*} \oplus d=$ $-d * \oplus d$. The first order special Lagrangian deformations (kernel of $F^{\prime}$ ) correspond to closed and co-closed 1-forms, i.e., harmonic 1-forms.

Next, to show that the deformation theory of special Lagrangian submanifolds is unobstructed, we call on the Banach space implicit function theorem. For this, we consider $F$ as a map from $C^{1, \alpha} 1$-forms to $C^{0, \alpha} n$ forms and 2-forms.

Lemma 3.12. $F$ is actually a map from $\Gamma(N(X))$ to exact $n$-forms and exact 2-forms.

Proof. It is obvious that the image of $F$ lies in the closed $n$-forms and 2forms, since $F$ is the pull back of the closed forms $\beta$ and $\kappa$. However, $\exp _{V}: X \rightarrow M$ is homotopic to the inclusion $i: X \rightarrow M$ by replacing $V$ with $t V$. Therefore, $\exp _{V}{ }^{*}$ and $i^{*}$ give the same map in cohomology. Thus, if $[\cdot]$ denotes cohomology class, then $\left[\exp _{V}{ }^{*}(\beta)\right]=\left[i^{*}(\beta)\right]=\left[\left.\beta\right|_{X}\right]=0$ since $X$ is special Lagrangian. Similarly, $\left[\exp _{V}^{*}(\kappa)\right]=0$. So the forms in the image of $F$ are cohomologous to zero, i.e. they are exact forms.

Hence, considering $F$ as a map from $C^{1, \alpha} 1$-forms to exact $C^{0, \alpha} n$-forms and 2-forms, then $F^{\prime}(0)=-d * \oplus d$ is surjective. The Banach space implicit function theorem says that $F^{-1}(0,0)$ is a manifold with tangent space at 0 
equal to kernel of $F^{\prime}=\mathcal{H}^{1}(X)$. Elliptic regularity implies that $F^{-1}(0,0)$ is, in fact, smooth.

Next, we have the following result which is the special Lagrangian analog of Simon's result for complex manifolds.

Theorem 3.13. Given a normal family of submanifolds $X_{t}$ with $X=X_{0}$ special Lagrangian and $V$ the normal vector field and $v=(J V)^{b}$ the corresponding 1-form, then

$$
\left.\frac{d^{2}}{d t^{2}} \operatorname{vol}\left(X_{t}\right)\right|_{t=0}=\int_{X}\left(\|d v\|^{2}+\left\|d^{*} v\right\|^{2}\right) d v o l_{0} .
$$

Corollary 3.14. Under the identification of the normal bundle with $T^{*}(X)$, the Jacobi operator is identified with the Hodge Laplacian $\Delta$.

Because the kernel of $\Delta$ is the same as the kernel of $d \oplus d^{*}$ on a compact manifold, we see the only way to deform a special Lagrangian submanifold as a minimal submanifold is to deform it as a special Lagrangian submanifold, although this is easily demonstrated since any minimal deformation of a calibrated submanifold is necessarily calibrated. However, the integrability of Jacobi fields of a general minimal submanifold is a difficult question, but in this context we have:

Corollary 3.15. Given a Jacobi vector field, one can realize it as a deformation vector field to a family of minimal submanifolds.

Proof of Theorem 3.13. We compute $\mathcal{Q}_{\alpha}(\nabla V)$. From the expansion of $\alpha$ :

$$
\begin{aligned}
\alpha= & \operatorname{Re}\left(\omega^{1}+i \omega^{n+1}\right) \wedge \cdots \wedge\left(\omega^{n}+i \omega^{2 n}\right) \\
= & \omega_{1} \wedge \cdots \wedge \omega_{n}-\left(\omega^{n+1} \wedge \omega^{n+2} \wedge \omega^{3} \wedge \cdots \wedge \omega^{n}+\cdots\right. \\
& \left.\quad+\omega^{1} \wedge \cdots \wedge \omega^{n-2} \wedge \omega^{2 n-1} \wedge \omega^{2 n}\right)+\cdots
\end{aligned}
$$

from which one can read off $\lambda_{1 \ldots k^{\prime} \ldots \ell^{\prime} \cdots p} \stackrel{j}{i}=-(1 / n !)\left(\delta_{k}^{i} \delta_{\ell}^{j}-\delta_{\ell}^{i} \delta_{k}^{j}\right)$. With these coefficients one can compute that

$$
\begin{aligned}
Q_{\alpha}(\nabla V) & =\sum_{i<j}-2\left(V_{i}^{i^{\prime}} V_{j}^{j^{\prime}}-V_{j}^{i^{\prime}} V_{i}^{j^{\prime}}\right) \\
& =-2 \sum_{i<j}\left(V_{i i} V_{j j}-V_{i j} V_{j i}\right) .
\end{aligned}
$$


Then one easily shows

$$
\|\nabla V\|^{2}-Q_{\alpha}(\nabla V)=\sum_{i, j}\left(V_{i j}\right)^{2}+2 \sum_{i<j}\left(V_{i i} V_{j j}-V_{i j} V_{j i}\right)
$$

agrees with

$$
\|d v\|^{2}+\left\|d^{*} v\right\|^{2}=\left(\sum V_{i i}\right)^{2}+\left(\sum_{i, j} V_{i j}-V_{j i}\right)^{2}
$$

and the result follows from the Theorem 1.4 for the second variation.

\section{Examples.}

(1) It is easy to write down flat complex $n$-dimensional tori equalling $\mathbb{C}^{n}$ mod various lattices with real $n$-dimensional sub-tori that are special Lagrangian. By the above results, the sub-tori translate in $n$ dimensional families.

(2) Since $S U(2) \cong S p(1)$, then if $M$ has an $S U(2)$ structure then $M$ has an $S p(1)$ structure. That is to say, $M$ will carry three distinct $S U(2)$ structures with complex structure maps $I, J$, and $K$ satisfying as usual $I^{2}=J^{2}=K^{2}=$-identity and the compatibility condition $I J=K$. It turns out that a surface is special Lagrangian with respect to one of the $S U(2)$ structures, say $I$ if and only if it is complex with respect to one of the other structures, in this case $K$. Thus, the special Lagrangian theory is equivalent to the complex theory. One can reinterpret the above special Lagrangian results as results in complex geometry. For example: There do not exist any rational curves (complex $S^{2}$ 's) on a complex 2-torus. A genus $g$ Riemann surface on either a complex 2-torus or a $\mathrm{K} 3$ surface will move (as a complex submanifold) in a real $2 g$ dimensional family.

(3) A particular result of the previous example is that if an elliptic curve $(g=1)$ lies on a K3 surface then this curve will move in a two dimensional family. Since the curve is of real codimension 2 , it can be hoped that the elliptic curves will foliate the K3 surface. In fact, the elliptic curves do foliate the $\mathrm{K} 3$ surface, if one allows certain degenerate leaves. 
(4) Suppose that on an $S U(n) M$, there is a real structure, i.e., an antiholomorphic involution $\tau$ such that the real points, i.e., those points fixed under the involution, form a smooth non-empty submanifold $X$. Then, in [Br2], Bryant shows that $X$ is special Lagrangian. For example,

$$
M=\left\{\left(z^{0}\right)^{n+2}+\cdots+\left(z^{n}\right)^{n+2}-\left(z^{n+1}\right)^{n+2}=0\right\} \subset \mathbb{C P}^{n+1}
$$

then the real structure $\tau\left(z^{i}\right)=\bar{z}^{i}$ of $\mathbb{C} \mathbb{P}^{n+1}$ restricts to $\tau: M \rightarrow M$. The real points consist of the subvariety

$$
X=\left\{\left(x^{0}\right)^{n+2}+\cdots+\left(x^{n}\right)^{n+2}-\left(x^{n+1}\right)^{n+2}=0\right\} \subset \mathbb{R} \mathbb{P}^{n+1},
$$

is a special Lagrangian submanifold of $M$. However, some care should be exercised since the induced metric is not the Calabi-Yau metric: Let

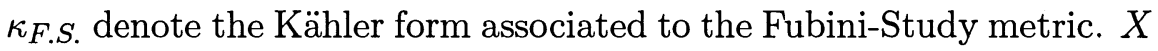
is Lagrangian with respect to the restriction to $M$ of the Fubini-Study metric, i.e., $\left.\kappa_{F . S .}\right|_{X}=0$. This is because the $\tau^{*}\left(\kappa_{F . S .}\right)=-\kappa_{F . S .}$ and $\tau$ is the identity on $X$. However, $X$ is also Lagrangian with respect to the Calabi-Yau metric on $M$. This is because the associated Kähler form $\kappa_{C . Y}$. on $M$ is $\left.\kappa_{F . S .}\right|_{M}+i \partial \bar{\partial} f$ with $f$ a real valued function on $M$ and

$$
\begin{aligned}
\tau^{*}\left(\left.i \partial \bar{\partial} f\right|_{X}\right) & =i \bar{\partial} \partial \tau^{*}\left(\left.f\right|_{X}\right) \\
& =-i \partial \bar{\partial} \tau^{*}\left(\left.f\right|_{X}\right)
\end{aligned}
$$

but $\tau$ is the identity on $X$, so, indeed, $\left.i \partial \bar{\partial} f\right|_{X}=0$.

For $n$ even, $X$ is homeomorphic to $S^{n}$ and $n$ odd $X$ is homeomorphic to $\mathbb{R} \mathbb{P}^{n}$. Explicit maps can be given as follows. For $n$ even, let $\left[y^{0}, \ldots, y^{n}\right]^{+}$be an oriented line in $\mathbb{R}^{n+1}$ with the space of all such lines being $\widetilde{\mathbb{R P}^{n}}=\left(\mathbb{R}^{n+1} \backslash 0\right) / \mathbb{R}^{+} \cong S^{n}$. We define the map from $\widetilde{\mathbb{R P}}{ }^{n}$ to $X$ via

$$
\left[y^{0}, \ldots, y^{n}\right]^{+} \mapsto\left[y^{0}, \ldots, y^{n},\left(\left(y^{0}\right)^{n}+\cdots+\left(y^{n}\right)^{n}\right)^{1 / n}\right] .
$$

For $n$ odd, we use the same map considered as a map from $\mathbb{R} \mathbb{P}^{n}$ to $X$. In both cases, it is easy to check that (3.9) provides the required homeomorphism. In both cases, too, we have $H^{1}(X)$ vanishes so that by the above results $X$ is rigid as a special Lagrangian manifold.

One can obtain Calabi-Yau manifolds by taking complete intersections in higher dimensional projective spaces, and by taking real slices one 
ought to find special Lagrangian manifolds that are not rigid although calculating the first Betti number of the real algebraic varieties would probably prove difficult.

\section{Deformations of Coassociative Submanifolds.}

We turn now to the deformation theory of coassociative submanifolds. This is analogous to the theory for special Lagrangian submanifolds, so we will try to parallel the previous section. Since coassociative submanifolds sit in seven dimensional manifolds with holonomy contained in $G_{2}$, we will first develop the structure equations for such manifolds $M^{7}$. We start with some constructions in flat space which will then be transferred to the tangent spaces of $M$.

On $\mathbb{R}^{7}$, define the associative 3 -form $\phi_{0}$ by:

$$
\phi_{0}=d x^{567}+d x^{5}\left(d x^{12}-d x^{34}\right)+d x^{6}\left(d x^{13}+d x^{24}\right)+d x^{7}\left(d x^{14}-d x^{23}\right)
$$

where $d x^{567}=d x^{5} \wedge d x^{6} \wedge d x^{7}$, etc.

Following [Br1], one defines $G_{2}$ to be the subgroup of $G L(7, \mathbb{R})$ fixing the form $\phi_{0}$. Fairly readily, one shows that $G_{2}$ preserves the metric given by:

$$
g_{0}=\left(d x^{1}\right)^{2}+\left(d x^{2}\right)^{2}+\left(d x^{3}\right)^{2}+\left(d x^{4}\right)^{2}+\left(d x^{5}\right)^{2}+\left(d x^{6}\right)^{2}+\left(d x^{7}\right)^{2} .
$$

Therefore, $G_{2}$ also preserves the cross product on $\mathbb{R}^{7}$ defined as follows: For $v, w \in \mathbb{R}^{7}$, then $v \times w$ equals $\left.\left.\sharp(w\lrcorner(v\lrcorner\left(\phi_{0}\right)\right)\right)$, where $\sharp:\left(\mathbb{R}^{7}\right)^{*} \rightarrow \mathbb{R}^{7}$ is the metric isomorphism. For example,

$$
\frac{\partial}{\partial x^{1}} \times \frac{\partial}{\partial x^{2}}=\frac{\partial}{\partial x^{5}} .
$$

With the cross product structure on $\mathbb{R}^{7}$, one easily defines an octonionic multiplication on $\mathbb{R}^{8}$; however, only the cross product structure will be needed.

Following [HL], let the coassociative 4-form $\psi_{0}$ be the Hodge dual to $\phi_{0}$. One calculates that

$$
\psi_{0}=d x^{1234}-d x^{67}\left(d x^{12}-d x^{34}\right)+d x^{57}\left(d x^{13}+d x^{24}\right)-d x^{56}\left(d x^{14}-d x^{23}\right)
$$

A 4-plane in $\mathbb{R}^{7}$ is defined to be coassociative if $\psi_{0}$ restricts to be the volume form. For example, the 4-plane

$$
\xi^{4}=\frac{\partial}{\partial x^{1}} \wedge \frac{\partial}{\partial x^{2}} \wedge \frac{\partial}{\partial x^{3}} \wedge \frac{\partial}{\partial x^{4}}
$$


is coassociative.

We identify $\mathbb{R}^{7}$ with $\mathbb{H} \oplus \operatorname{Im} \mathbb{H}$ by

$$
\left(x^{1}, \ldots, x^{7}\right) \mapsto\left(x^{1}+i x^{2}+j x^{3}+k x^{4}, i x^{5}+j x^{6}+k x^{7}\right)=(u, v),
$$

and let

$$
[p, q] \in S p(1) \times S p(1) /\{ \pm(1,1)\}=S O(4)
$$

act on $\mathbb{R}^{7}=\mathbb{H} \oplus \operatorname{Im} \mathbb{H}$ via: for $u \in \mathbb{H}$ then $u \mapsto p u \bar{q}$ and for $v \in \operatorname{Im} \mathbb{H}$ then $v \mapsto q v \bar{q}$. This action of $S O(4)$ on $\mathbb{R}^{7}$ is just the sum of the standard representation of $S O(4)$ on $\mathbb{R}^{4}$ together with the anti-self dual representation on $\Lambda_{-}^{2}\left(\mathbb{R}^{4}\right) \cong \mathbb{R}^{3}$. $S O(4)$ preserves the subspace

$$
\mathbb{H}=\frac{\partial}{\partial x^{1}} \wedge \frac{\partial}{\partial x^{2}} \wedge \frac{\partial}{\partial x^{3}} \wedge \frac{\partial}{\partial x^{4}},
$$

and also $S O(4)$ preserves the the associative 3 -form $\phi_{0}$. This last statement is seen by writing $\phi_{0}$ as

$$
\phi_{0}=-\frac{1}{6} \nu_{0} \wedge \nu_{0} \wedge \nu_{0}-\frac{1}{2} \operatorname{Re}\left(\nu_{0} \wedge \bar{\mu}_{0} \wedge \mu_{0}\right)
$$

where $\mu_{0}=d x^{1}+i d x^{2}+j d x^{3}+k d x^{4}$ and $\nu_{0}=i d x^{5}+j d x^{6}+k d x^{7}$. We then have

Proposition 4.1. $G_{2}$ acts transitively on the set of coassociative 4-planes with isotropy $S O(4)$.

Proof. See [HL], Theorem 1.8, Chapter IV.

Next, we place these structures on the tangent space to a seven manifold $M$. We say that $M$ has an almost $G_{2}$ structure if $M$ carries a positive 3 -form $\phi$. Here positive just means there is some identification of each tangent space to $\mathbb{R}^{7}$ taking $\phi$ to $\phi_{0}$. Via such an identification, one may pull back the metric $g_{0}$ to each tangent space, giving a well-defined Riemannian metric on $M$. The almost $G_{2}$ structure is said to be integrable if the form $\phi$ is parallel (covariant constant) with respect to the corresponding Levi-Civita connection. In $[\mathrm{Br} 1]$, it is shown that integrability is equivalent to the closure and co-closure of the form $\phi$. Let us call such manifolds with integrable $G_{2}$ structures, $G_{2}$ manifolds, and we assume $M$ to be such a manifold.

We now define the $G_{2}$ coframe bundle of $M^{7}, \mathcal{F}(M)$, as follows. For $p \in M$ let $\mathcal{F}_{p}$ be $\left\{u: T_{p}(M) \rightarrow \mathbb{R}^{7} \mid u^{*} \phi_{0}=\phi\right\} . \mathcal{F}_{p} \neq \emptyset$ by our definition of 
positivity of $\phi$, and so by our definition of $G_{2}, \mathcal{F}_{p} \cong G_{2}$. There is a canonical $\mathbb{R}^{7}$-valued 1-form on $\mathcal{F}$ defined by $\omega(V)=u \circ \pi_{*}(V)$ where $\pi$ denotes the projection of $\mathcal{F}$ to $M$ and $V \in T_{u}(\mathcal{F})$. With this the structure equations take the form:

$$
d\left(\begin{array}{c}
\omega^{i} \\
\omega^{a}
\end{array}\right)=-\left(\begin{array}{cc}
\varphi_{j}^{i} & \beta_{b}^{i} \\
\beta_{j}^{a} & \rho_{-}(\varphi)_{b}^{a}
\end{array}\right)\left(\begin{array}{l}
\omega^{j} \\
\omega^{b}
\end{array}\right)
$$

where $1 \leqslant i, j, k, \cdots \leqslant 4$ and $1 \leqslant a, b, c, \cdots \leqslant 3, \varphi_{j}^{i}=-\varphi_{i}^{j}, \beta_{i}^{a}=-\beta_{a}^{i}$, and $\rho_{-}: \mathfrak{s o}(4) \rightarrow \mathfrak{s o}(3)$ denotes the anti-self dual representation. Also, $\beta$ is not arbitrary, but if one thinks of the three columns of $\beta_{a}^{i}$ as quaternions $\beta_{1}, \beta_{2}$ and $\beta_{3}$ then they satisfy the relation $i \beta_{1}+j \beta_{2}+k \beta_{3}=0$. (Integrability ensures that there is no torsion.)

One has $\phi$ and $\psi$ (pulled up to $\mathcal{F}$ ) given by:

$$
\phi=\omega^{567}+\omega^{5} \wedge\left(\omega^{12}-\omega^{34}\right)+\omega^{6} \wedge\left(\omega^{13}+\omega^{24}\right)+\omega^{7} \wedge\left(\omega^{14}-\omega^{23}\right)
$$

and

$$
\psi=\omega^{1234}-\omega^{67} \wedge\left(\omega^{12}-\omega^{34}\right)+\omega^{57} \wedge\left(\omega^{13}+\omega^{24}\right)-\omega^{56} \wedge\left(\omega^{14}-\omega^{23}\right)
$$

A four dimensional submanifold $X^{4} \subset M^{7}$ is said to be coassociative if each tangent space is coassociative, that is the 4 -form $\psi$ restricts to $X$ to be the volume form. Now if $X^{4}$ is a coassociative submanifold of $M^{7}$, we may pull back the $G_{2}$ coframe bundle of $M$ and take the first adapted bundle $\mathcal{F}^{(1)}\left(X^{4}\right)$ where the $\omega^{a}$ 's restricts to zero and the $\omega^{i}$ 's are semibasic for the projection to $X$. By the previous proposition, this is a principal $S O(4)$ bundle over $X$. For coassociative submanifolds, we have the following result.

Proposition 4.2. The normal bundle to a coassociative submanifold is isomorphic to the bundle of anti-self dual 2-forms, i.e., $N(X) \cong \Lambda_{-}^{2}(X)$.

Proof. This result follows just from the fact that both bundles arise as vector bundles associated to the principal bundle $\mathcal{F}^{(1)}\left(X^{4}\right)$ via the same representation $\rho_{-}: S O(4) \rightarrow G L(\operatorname{Im} \mathbb{H})$, namely, $\rho_{-}([p, q])(x)=\bar{q} x q$ for $x \in \operatorname{Im} \mathbb{H}$.

One can give an explicit map from $\Lambda_{-}^{2}(X)$ to $N(X)$. For $\alpha \in \Lambda_{-}^{2}(X)$, choose an orthonormal basis $e_{i}$ of $T_{p}(X)$, such that $\alpha=A\left(\mu_{1} \mu_{2}-\mu_{3} \mu_{4}\right)$ where $A$ is a scalar and the $\mu_{i}$ 's are dual to the $e_{i}$ 's. Send $\alpha$ to $A e_{1} \times e_{2}$. To show this map is well-defined and normal, we may use the $G_{2}$ invariance and identify $T_{p}(X)$ with $\xi^{4}$ as in (4.5). To show the map is well defined, one notes that the isotropy in $S O(4)$ of $\omega^{12}-\omega^{34}$ is a $U(2)=\left\{\left[e^{i \theta}, q\right] \mid \theta \in\right.$ 
$\mathbb{R}, q \in S p(1)\}$, and this $U(2)$ fixes $e_{1} \times e_{2}$. By (4.3), the map is normal valued.

Let $\alpha^{1}=\omega^{12}-\omega^{34}, \alpha^{2}=\omega^{13}+\omega^{24}$, and $\alpha^{1}=\omega^{14}-\omega^{23}$. The general normal vector field

$$
V=V^{5} \frac{\partial}{\partial \omega^{5}}+V^{6} \frac{\partial}{\partial \omega^{6}}+V^{7} \frac{\partial}{\partial \omega^{7}}
$$

is identified with the anti-self dual 2-form $\alpha_{V}=V_{1} \alpha^{1}+V_{2} \alpha^{2}+V_{3} \alpha^{3}$ where we set $V_{1}=V^{5}, V_{2}=V^{6}, V_{3}=V^{7}$.

Corollary 4.3. For a coassociative submanifold $X$ of a flat seven torus, $T^{7}$, with a $G_{2}$ structure, then (1) $T(X) \oplus \Lambda_{-}^{2}(X)$ is trivial, and so (2) $p_{1}(M)+p_{1}\left(\Lambda_{1}^{2}(X)\right)=p_{1}(X)+p_{1}(X)+2 e(X)=0$, and therefore (3) $3 \chi(X)+\sigma(X)=0$ where $\chi$ and $\sigma$ are the Euler characteristic and signature, respectively.

Proof. The first statement follows from the fact the tangent bundle to the torus is trivial, and so the restriction to $X$ is trivial, and $\left.T(M)\right|_{X} \cong$ $T(X) \oplus \Lambda_{-}^{2}(X)$. The second statement follows from the fact that $p_{1}\left(\Lambda_{-}^{2}(X)\right)$ equals $p_{1}(X)+2 e(X)$, which follows from an easy classifying space argument. The third follows from the signature theorem for four manifolds: $\sigma(X)=\frac{1}{3} \int_{X} p_{1}(X)$.

Next, we turn to the deformation theory of coassociative submanifolds. For this we need the following reformulation of coassociativity.

Proposition 4.4. A four dimensional submanifold is coassociative if and only if the 3-form $\phi$ restricts to $X$ to be zero.

Proof. See [HL], Corollary 1.20, Chapter IV.

With these preliminaries we have

Theorem 4.5. A normal vector field to a compact smooth coassociative submanifold is the deformation vector field to a family of coassociative submanifolds if and only if the corresponding 2-form is closed (and hence coclosed by anti-self duality) hence harmonic. Thus the Zariski tangent space to the moduli space of coassociative submanifolds at $X$ is isomorphic to $\mathcal{H}_{-}^{2}(X)$. Furthermore, there are no obtructions. 
Corollary 4.6. If $\beta_{-}^{2}(X)=\operatorname{dim} H_{-}^{2}(X, \mathbb{R})$ is zero, then $X$ is rigid as a coassociative submanifold.

More generally:

Corollary 4.7. The moduli space of coassociative submanifolds is locally a smooth manifold of dimension equalling $\beta_{-}^{2}(X)$. Furthermore, the moduli space carries a natural Riemannian metric: Given two tangent vectors, identify them with anti-self dual forms $\alpha$ and $\alpha^{\prime}$ on $X$, then their inner product is defined to be $-\int_{X} \alpha \wedge \alpha^{\prime}$.

Let us recall some of these dimensions for familiar four manifolds:

$\begin{array}{cc}X & \beta_{-}^{2}(X) \\ S^{4} & 0 \\ \mathbb{C P}^{2} & 0 \\ \overline{\mathbb{C P}^{2}} & 1 \\ T^{4} & 3 \\ K 3 & 19 \\ \overline{K 3} & 3\end{array}$

Some particular cases: If the four sphere or $\mathbb{C P}^{2}$ occurs as a coassociative submanifold, then it will be rigid. If $\overline{\mathbb{C P}^{2}}$ occurs, it will move in a one dimensional family. The four torus would move in a three dimensional family, etc.

The most intriguing case is, if a $\overline{K 3}$ occurs it would move in a three dimensional family. Since it would be of codimension three in $M$, one may hope that the family would fill up the whole ambient manifold, possibly with some degenerations.

Corollary 4.8. A coassociative submanifold of a flat torus with $G_{2}$ structure has $\beta_{-}^{2}(X) \geq 3$.

Proof of Corollary 4.8. The seven killing vector fields on the torus will project to at least three linearly independent normal vector fields on the submanifolds giving rise to three distinct families of coassociative submanifolds.

This result, with the restriction on the Euler character and signature from Corollary 4.3, severely restricts the topology of coassociative submanifolds of $G_{2}$ tori. 
Proof of Theorem 4.5. We define a non-linear map $F: U \subset N(X) \rightarrow \Omega^{3}(X)$ by pulling back the form $\phi$ via the map $\exp _{V}$. By Proposition $4.4, F^{-1}(0)$ is the space of nearby coassociative submanifolds. Again we need to compute the linearization of $F$ :

$$
\begin{aligned}
F^{\prime}(0)(V) & =\left.\frac{\partial}{\partial t} F(t V)\right|_{t=0} \\
& =\left.\mathcal{L}_{V}(\psi)\right|_{X} \\
& =(V\lrcorner d \psi+d(V\lrcorner \psi))\left.\right|_{X} \\
& =d(V\lrcorner \psi)\left.\right|_{X} .
\end{aligned}
$$

From (4.8), we find

$$
\left.V\lrcorner\left.\psi\right|_{X}=\left(V^{a} \frac{\partial}{\partial \omega^{a}}\right)\right\lrcorner\left.\psi\right|_{X}=V_{1} \alpha^{1}+V_{2} \alpha^{2}+V_{3} \alpha^{3}=\alpha_{V},
$$

where $\alpha_{V}$ is the anti-self dual 2-form corresponding to the normal vector field. (See Proposition 4.2.) Thus $F^{\prime}(0)(V)=d \alpha_{V}$, and so $F^{\prime}(0)$ is just exterior differentiation when $F^{\prime}(0)$ is considered as a map $\Omega_{-}^{2}(X) \rightarrow \Omega^{3}(X)$. The kernel is the harmonic anti-self dual 2-forms. (Recall closed and antiself dual implies co-closed, hence harmonic.) This proves the Zariski tangent space is $\mathcal{H}_{-}^{2}(X)$.

To show that it is unobstructed, we view $F$ as a map of anti-self dual 2 -forms of class $C^{1, \alpha}$ to 3 -forms of class $C^{0, \alpha}$. One notes that $F$ actually maps to those 3 -forms cohomologous to zero. The reasoning is the same as in the special Lagrangian case, see Lemma 3.11. Thus, via standard Hodge theory, $F: \Omega_{-}^{2}(X) \rightarrow d \Omega_{-}^{2}(X) \subset \Omega^{3}(X)$. Hence $F^{\prime}(0)=d$ is surjective, so the Banach space implicit function theorem asserts that $F^{-1}(0)$ is a smooth manifold with tangent space at 0 equal to the kernel of $F^{\prime}(0)$, i.e., $\mathcal{H}_{-}^{2}(X)$.

Next, we turn to the second variation of volume of a coassociative submanifold.

Theorem 4.9. For a normal family of submanifolds $X_{t}$ with $X=X_{0}$ coassociative, $V=\frac{\partial}{\partial t} X_{t}$ the deformation vector field, and $\alpha_{V}$ the corresponding anti-self dual 2-form, then

$$
\frac{d^{2}}{d t^{2}} \operatorname{vol}\left(X_{t}\right)=\int_{X}\left\langle d \alpha_{V}, d \alpha_{V}\right\rangle
$$


Corollary 4.10. Under the identification of the normal bundle with $\Lambda_{-}^{2}(X)$, the Jacobi operator is identified with the Hodge Laplacian $\Delta$.

Because the kernel of $\Delta: \Omega_{-}^{2}(X) \rightarrow \Omega_{-}^{2}(X)$ is the same as the kernel of $d: \Omega_{-}^{2}(X) \rightarrow \Omega^{3}(X)$ on a compact four dimensional manifold, we see the only way to deform a coassociative submanifold as a minimal submanifold is to deform it as a coassociative submanifold. Again, this follows from the fact that minimal deformations of a calibrated submanifold are all calibrated. We have the analog of Corollary 3.15

Corollary 4.11. Given a Jacobi vector field, one can realize it as a deformation vector field to a family of minimal submanifolds, i.e., all Jacobi fields are integrable.

Proof of Theorem 4.9. Using the formula of the second variation of calibrated submanifolds, one only needs to calculate $\mathcal{Q}_{\psi}(\nabla V)$. If

$$
d V^{a}+\rho_{-}(\varphi)_{b}^{a} V^{b}=V_{i}^{a} \omega^{i},
$$

where $V_{i}^{a}$ are the components of the covariant derivative of $V$, then using equation (4.8), we read off the coefficients $\lambda_{6734}=-1 / 4$ !, etc. From which, we compute

$$
\begin{aligned}
\mathcal{Q}_{\psi}(\nabla V)=2 & V_{2}^{6} V_{1}^{7}-V_{1}^{6} V_{2}^{7}+V_{3}^{6} V_{4}^{7}-V_{4}^{6} V_{3}^{7} \\
& +V_{1}^{5} V_{3}^{7}-V_{3}^{5} V_{1}^{7}+V_{2}^{5} V_{4}^{7}-V_{4}^{5} V_{2}^{7} \\
& \left.-V_{1}^{5} V_{4}^{6}+V_{4}^{5} V_{1}^{6}-V_{3}^{5} V_{2}^{6}+V_{2}^{5} V_{3}^{6}\right) \\
=2 & \left(V_{22} V_{31}-V_{21} V_{32}+V_{23} V_{34}-V_{24} V_{33}\right. \\
& +V_{11} V_{33}-V_{13} V_{31}+V_{12} V_{34}-V_{14} V_{32} \\
& \left.-V_{11} V_{24}+V_{14} V_{21}-V_{13} V_{22}+V_{12} V_{23}\right) .
\end{aligned}
$$

Since

$$
\begin{aligned}
d \alpha_{V}= & \left(V_{1 i} \omega^{i}\right)\left(\omega^{12}-\omega^{34}\right)+\left(V_{2 i} \omega^{i}\right)\left(\omega^{13}+\omega^{24}\right) \\
& +\left(V_{3 i} \omega^{i}\right)\left(\omega^{14}-\omega^{23}\right) \\
= & \left(V_{13}-V_{22}-V_{31}\right) \omega^{123}+\left(V_{14}+V_{21}-V_{32}\right) \omega^{124} \\
& +\left(V_{24}-V_{11}-V_{33}\right) \omega^{134}-\left(V_{12}+V_{23}+V_{34}\right) \omega^{234}
\end{aligned}
$$

it is easy to show that

$$
\|\nabla V\|^{2}-\mathcal{Q}_{\psi}(\nabla V)=\left\langle d \alpha_{V}, d \alpha_{V}\right\rangle .
$$


so the theorem follows from Theorem 2.4.

Examples. This section is somewhat short, due to the fact that so very few examples of $G_{2}$ manifolds are known. In fact, one of the prime motivations for studying calibrated submanifolds is hopefully to shed some light on the ambient manifold.

(1) The first example is the case of just the flat torus given by $\mathbb{R}^{7}=$ $\mathbb{H} \oplus \operatorname{Im} \mathbb{H}$ modulo a lattice $\Lambda$ and a coassociative subtorus. By the above results, the subtorus will move in only the obvious directions. Such tori and subtori certainly exist, for example, $\Lambda=\Lambda_{1} \oplus \Lambda_{2}$ where $\Lambda_{1} \subset \mathbb{H}$ and $\Lambda_{2} \subset \operatorname{Im} \mathbb{H}$ results the sub-torus moves in a three dimensional family. Also, it only moves in the three dimensional family.

(2) If one takes the product of a $K 3$ surface with its Calabi-Yau metric and a flat three torus, then the total space carries a $G_{2}$ structure for which the $K 3 \times\{$ pt. $\}$ are coassociative. They, again, only move in the obvious 3 -dimensional family.

(3) In [BS], Bryant and Salamon construct $G_{2}$ structures on the total space of rank three bundles (in fact $\Lambda_{-}^{2}$ ) over the four-sphere and $\mathbb{C P}^{2}$ where the zero section is a coassociative submanifolds of the total space. By the above results they are rigid.

\section{Deformations of Associative Submanifolds.}

In this section, we turn to the associative submanifolds of manifolds with holonomy $G_{2}$. Associative submanifolds are those three manifolds $X$ for which the 3 -form $\phi$, of the previous section, restricts to be the volume form. Hence, we will follow the notation of the previous section with the exceptions: the rôles of the tangential and normal variables are reversed. Accordingly, we relabel so that the associative 3 -form $\phi$ is given by:

$$
\phi=\omega^{123}+\omega^{1} \wedge\left(\omega^{45}-\omega^{67}\right)+\omega^{2} \wedge\left(\omega^{46}+\omega^{57}\right)+\omega^{3} \wedge\left(\omega^{47}-\omega^{56}\right) .
$$

In this section, the index ranges are: $1 \leq i, j, k \leq 3$ and $4 \leq a, b, c, d \leq 7$.

Given an associative three dimensional submanifold $X$ in $M^{7}$, we pull back the $G_{2}$ coframe bundle and then take the adapted frame bundle, $\mathcal{F}^{(1)}(X)$ where $\eta$ restricts to zero. As in the previous section, this is a principal right $S O(4)$ bundle. We collect the following facts, most of whose proofs may be found in the text by Lawson and Michelson [LM]: 
(1) The tangent bundle of $X$ is the vector bundle associated to the principal bundle $\mathcal{F}^{(1)}(X)$ via the representation

$$
\rho_{-}: S O(4) \rightarrow G L(\operatorname{Im} \mathbb{H})
$$

where $\rho_{-}([p, q])(x)=q x \bar{q}$ for all $x \in \operatorname{Im} \mathbb{H} \cong \mathbb{R}^{3}$, and

$$
[p, q] \in S O(4)=S p(1) \times S p(1) /\{ \pm(1,1)\} .
$$

Tangent vector fields are given by equivariant maps $W: \mathcal{F}^{(1)}(X) \rightarrow$ $\operatorname{Im} \mathbb{H}$ where $R_{[p, q]}^{*}(W)=\bar{q} W q$.

(2) Oriented three manifolds are always spinnable. A choice of a spin structure on $X$ determines an $S p(1) \times S p(1)$ bundle $\mathcal{P}$ which double covers $\mathcal{F}^{(1)}(X)$. The spin bundle $\mathbb{S}$ of $X$ is associated to the principal bundle $\mathcal{P}$ via the representation

$$
\sigma: S p(1) \times S p(1) \rightarrow G L(\mathbb{H})
$$

where $\sigma(p, q)(y)=y \bar{q}$ for $y \in \mathbb{H}$. Spinors are given by maps from $S: \mathcal{P} \rightarrow \mathbb{H}$ such that $R_{(p, q)}^{*}(S)=S q$.

(3) The normal bundle is the vector bundle associated to $\mathcal{F}^{(1)}(X)$ via the representation $\tau: S O(4) \rightarrow G L(\mathbb{H})$ where $\tau([p, q])(y)=p y \bar{q}$ for $y \in \mathbb{H}$. Normal vector fields, $V_{a} \frac{\partial}{\partial \eta_{a}}$, are given by maps $V: \mathcal{F}^{(1)}(X) \rightarrow$ $\mathbb{H}$ satisfying $R_{[p, q]}^{*}(V)=\bar{p} V q$. Alternatively, the normal bundle is associated to $\mathcal{P}$ via the representation

$$
\tilde{\tau}: S p(1) \times S p(1) \rightarrow G L(\mathbb{H})
$$

where $\tilde{\tau}(p, q)(y)=p y \bar{q}$ for $y \in \mathbb{H}$.

(4) Let us denote the vector bundle associated to $\mathcal{P}$ via the representation $\varepsilon(p, q)(y)=\bar{p} y$ by $E$. We see that the normal bundle is $\mathbb{S} \otimes_{\mathbb{H}} E$.

(5) If $V=V^{a} \frac{\partial}{\partial \omega^{a}}$ is a normal vector field, and

$$
d V^{a}+\varphi_{b}^{a} V^{b}=V_{i}^{a} \omega_{i}
$$

so that $\nabla(V)=V_{i}^{a} \frac{\partial}{\partial \omega^{a}} \otimes \omega_{i}$, then, viewing $V$ as a twisted spinor:

$$
V=V^{4}+i V^{5}+j V^{6}+k V^{7}
$$


we have:

$$
\begin{aligned}
\not D(V)=- & \left(V_{1}^{5}+V_{2}^{6}+V_{3}^{7}\right)+i\left(V_{1}^{4}+V_{3}^{6}-V_{2}^{7}\right) \\
& +j\left(V_{2}^{4}-V_{3}^{5}+V_{1}^{7}\right)+k\left(V_{3}^{4}-V_{2}^{5}-V_{1}^{6}\right) .
\end{aligned}
$$

Recall in dimension three the Dirac operator is given by:

$$
\not D=i \nabla_{1}+j \nabla_{2}+k \nabla_{3} .
$$

With these remarks at hand, we turn to the deformation theory of associative three manifolds. Again, we call on an alternative characterization of associative submanifolds found in [HL]. There it is shown that $\operatorname{Im} \mathbb{O} \cong \mathbb{R}^{7}$ carries a triple cross product that is invariant under $G_{2}$. The cross product may be transferred to the tangent spaces of a $G_{2}$ manifold, and can be thought of as a differential 3-form $\chi$ taking values in the tangent space, i.e., $\chi$ is an element of $\Omega^{3}(M, T(M))$. One can compute that

$$
\begin{aligned}
\chi= & \left(\omega^{256}-\omega^{247}+\omega^{346}+\omega^{357}\right) \frac{\partial}{\partial \omega^{1}} \\
& +\left(\omega^{156}-\omega^{147}-\omega^{345}+\omega^{367}\right) \frac{\partial}{\partial \omega^{2}} \\
& +\left(\omega^{245}-\omega^{267}-\omega^{146}-\omega^{157}\right) \frac{\partial}{\partial \omega^{3}} \\
& +\left(\omega^{567}-\omega^{127}+\omega^{136}-\omega^{235}\right) \frac{\partial}{\partial \omega^{4}} \\
& +\left(\omega^{126}-\omega^{467}+\omega^{137}+\omega^{234}\right) \frac{\partial}{\partial \omega^{5}} \\
& +\left(\omega^{457}-\omega^{127}-\omega^{134}+\omega^{237}\right) \frac{\partial}{\partial \omega^{6}} \\
& +\left(\omega^{124}-\omega^{456}-\omega^{135}-\omega^{236}\right) \frac{\partial}{\partial \omega^{7}}
\end{aligned}
$$

Proposition 5.1. A three dimensional submanifold is associative if and only if the form $\chi$ restricts to be zero.

Proof. See [HL], Corollary 1.19, Chapter IV.

With these preliminaries, we can finally state the following result:

Theorem 5.2. Under the correspondence of normal vector fields with twisted spinors, the Zariski tangent space to associative submanifolds at an 
associative submanifold $X$ is the space of harmonic twisted spinors on $X$, that is the kernel of the twisted Dirac operator. In other words, the condition on a normal vector field that it be the deformation vector field to first order to a family of associative submanifolds is that the normal vector field should correspond to a harmonic spinor in the isomorphism $N(X) \cong \mathbb{S} \otimes_{\mathbb{H}} E$.

Proof. We define the nonlinear map $F$ on a neighborhood $U$ of $0 \in \Gamma(N(X))$, i.e., small normal vector fields. $F(V)$ is defined to be the pull back of $\chi$ using the map $\exp _{V} . F$ takes values in

$$
\Omega^{3}(X, f *(T(M)))=\Omega^{3}(X, f *(T(X))) \oplus \Omega^{3}(X, f *(N(X))) .
$$

By Proposition 5.1, $F^{-1}(0)$ corresponds to nearby associative submanifolds. As in the previous sections, we compute:

$$
\begin{aligned}
F^{\prime}(0)(V) & =\left.\frac{\partial}{\partial t} F(t V)\right|_{t=0} \\
& =\left.\mathcal{L}_{V}(\chi)\right|_{X} \\
& =(V\lrcorner d \chi+d(V\lrcorner \chi))\left.\right|_{X} \\
& =d(V\lrcorner \chi)\left.\right|_{X} .
\end{aligned}
$$

From (5.4), we find

$$
\begin{aligned}
F^{\prime}(0)(V)=( & -\left(V_{1}^{5}+V_{2}^{6}+V_{3}^{7}\right) \frac{\partial}{\partial \omega^{4}} \\
& +\left(V_{1}^{4}+V_{3}^{6}-V_{2}^{7}\right) \frac{\partial}{\partial \omega^{5}} \\
& +\left(V_{2}^{4}-V_{3}^{5}+V_{1}^{7}\right) \frac{\partial}{\partial \omega^{6}} \\
& \left.+\left(V_{3}^{4}-V_{2}^{5}-V_{1}^{6}\right) \frac{\partial}{\partial \omega^{7}}\right) \otimes \omega^{123}
\end{aligned}
$$

Thus comparing (5.2) and (5.6), we see that the infinitessimal associative deformations (normal vector fields in the kernel of $F^{\prime}(0)$ ) are in one to one correspondence with harmonic spinors.

Remarks. The situation here contrasts greatly with the previous two calibrations. On an odd dimensional manifold, the index of any elliptic operator vanishes. In particular, the index of the Dirac operator vanishes. Unfortunately, one has no control on the dimension of the kernel, just that it will 
be matched by the dimension of the cokernel. Also, trying to decide unobstructedness, seems very difficult since, as soon as there are deformations, there is a non-zero obstruction space, essentially the cokernel.

Turning now to the second variation for associative submanifolds, we have the following:

Theorem 5.3. Given a normal deformation $X_{t}$ of $X=X_{0}$ with $X$ associative and $V=\left.\frac{\partial}{\partial t} X_{t}\right|_{t=0}$ the deformation vector field, then

$$
\left.\frac{d^{2}}{d t^{2}} \operatorname{vol}\left(X_{t}\right)\right|_{t=0}=2 \int_{X}\langle\not D(V), \not D(V)\rangle d v o_{0}
$$

Corollary 5.4. Under the identification of the normal bundle with the twisted spin bundle the Jacobi operator is identified with the Dirac Laplacian $\not D^{2}$.

Because the kernel of $\not \supset$ is the same as the kernel of $\not \not^{2}$ on a compact manifold, we reduce the integrability question of Jacobi fields to the above (difficult) integrability problem.

Proof of Theorem 5.3. Reading off the necessary coefficients from our expression (5.1) for $\phi$, one calculates

$$
\begin{aligned}
\mathcal{Q}_{\phi}(\nabla V)=2 & \left(V_{2}^{4} V_{3}^{5}-V_{3}^{4} V_{2}^{5}+V_{3}^{6} V_{2}^{7}-V_{2}^{6} V_{3}^{7}\right. \\
& +V_{3}^{4} V_{1}^{6}-V_{1}^{4} V_{3}^{6}+V_{3}^{5} V_{1}^{7}-V_{1}^{5} V_{3}^{7} \\
& \left.+V_{1}^{4} V_{2}^{7}-V_{2}^{4} V_{1}^{7}-V_{1}^{5} V_{2}^{6}+V_{2}^{5} V_{1}^{6}\right),
\end{aligned}
$$

Now from formula (5.2) for $\not D(V)$, it is a matter of algebra to compute that

$$
\|\nabla V\|^{2}-\mathcal{Q}_{\phi}(\nabla V)=\langle\not D(V), \not D(V)\rangle .
$$

and the theorem follows from Theorem 1.4.

Examples. Aside from the flat examples, Bryant and Salamon ([BS]) construct a metric with holonomy $G_{2}$ on (an open set of) the total space of the spin bundle $\mathbb{S}$ of a constant curvature three manifold $X$. The zero section (which we identify with $X$ ) is associative. It is clear that the normal bundle to the zero section is just the spin bundle $\mathbb{S}$, itself. Hence, the bundle $E$ defined in the fourth remark is trivial, and the Zariski tangent space at 
$X$ to the moduli of associative submanifolds is isomorphic to the harmonic spinors rather than harmonic twisted spinors.

There are three cases depending on the sign of the scalar curvature $S$ of $X$. For $S>0$, there are no harmonic spinors by the vanishing theorem of Lichnerowicz, [Li], so the zero section is rigid. For $S=0$, the zero section is trivially deformable since the spin bundle is flat. For $S<0$, there may be examples of non-trivial deformations.

\section{Deformations of Cayley Submanifolds.}

Since Cayley submanifolds lie in manifolds with holonomy contained in Spin (7), we need to now give the structure equations for Spin (7) manifolds.

We begin by giving Bryant's unusual definition of Spin(7), [Br1]. On $\mathbb{R}^{8}$, define the Cayley 4 -form to be:

$$
\begin{aligned}
\Phi_{0}= & d x^{1234}+\left(d x^{12}-d x^{34}\right) \wedge\left(d x^{56}-d x^{78}\right) \\
& +\left(d x^{13}+d x^{24}\right) \wedge\left(d x^{57}+d x^{68}\right) \\
& +\left(d x^{14}-d x^{23}\right) \wedge\left(d x^{58}-d x^{67}\right)+d x^{5678}
\end{aligned}
$$

One then defines Spin (7) to be the subgroup of $G L(8)$ that preserves $\Phi_{0}$. In $[\mathrm{Br} 1]$, it is shown that this definition corresponds to the usual one, i.e., the simply connected cover of $S O(7)$. Also, it is shown there that $\operatorname{Spin}(7)$ preserves the metric on $\mathbb{R}^{8}$ equal to

$$
g_{0}=\left(d x^{1}\right)^{2}+\left(d x^{2}\right)^{2}+\left(d x^{3}\right)^{2}+\left(d x^{4}\right)^{2}+\left(d x^{5}\right)^{2}+\left(d x^{6}\right)^{2}+\left(d x^{7}\right)^{2}+\left(d x^{8}\right)^{2} .
$$

A 4-plane $\xi$ in $\mathbb{R}^{8}$ is said to be a Cayley 4-plane if the form $\Phi_{0}$ restricts to be the volume form. For example, the 4-plane

$$
\xi^{4}=\frac{\partial}{\partial x^{1}} \wedge \frac{\partial}{\partial x^{2}} \wedge \frac{\partial}{\partial x^{3}} \wedge \frac{\partial}{\partial x^{4}},
$$

is Cayley.

We identify $\mathbb{R}^{8}$ with $\mathbb{H} \oplus \mathbb{H}$ by

$$
\left(x^{1}, \ldots, x^{8}\right) \mapsto\left(x^{1}+i x^{2}+j x^{3}+k x^{4}, x^{5}+i x^{6}+j x^{7}+k x^{8}\right)=(u, v),
$$

and let $([p, q, r]) \in S p(1) \times S p(1) \times S p(1) /\{ \pm(1,1,1)\}=H$ act on $\mathbb{R}^{8}=\mathbb{H} \oplus \mathbb{H}$ via: for $(u, v) \in \mathbb{H} \oplus \mathbb{H}$ then $(u, v) \mapsto(p u \bar{q}, r v \bar{q})$. The group $H$ preserves the decomposition $\mathbb{R}^{8}=\mathbb{H} \oplus \mathbb{H}$, and so also the Cayley plane $\xi$ in (6.3). The 
subgroup $H$ also preserves the Cayley 4 -form. This may be seen by writing $\Phi_{0}$ as

$$
\Phi_{0}=-\frac{1}{4}\left(\bar{\mu}_{0} \wedge \mu_{0} \wedge \bar{\mu}_{0} \wedge \mu_{0}+\bar{\mu}_{0} \wedge \mu_{0} \wedge \bar{\nu}_{0} \wedge \nu_{0}+\bar{\nu}_{0} \wedge \nu_{0} \wedge \bar{\nu}_{0} \wedge \nu_{0}\right)
$$

where $\mu_{0}=d x^{1}+i d x^{2}+j d x^{3}+k d x^{4}$ and $\nu_{0}=d x^{5}+i d x^{6}+j d x^{7}+k d x^{8}$. Thus $H$ is contained in the isotropy subgroup of $\operatorname{Spin}(7)$ preserving the Cayley plane $\xi$. The converse is true also, and so we have:

Proposition 6.1. Spin (7) acts transitively on the set of Cayley 4-planes with isotropy $H=S p(1) \times S p(1) \times S p(1) /\{ \pm(1,1,1)\}$.

Proof. See [HL], Theorem 1.8, Chapter IV.

We now turn to definition of Spin(7) manifolds. An eight dimensional manifold $M$ has an almost $\operatorname{Spin}(7)$ structure if it carries a nondegenerate 4-form, where a 4-form is nondegenerate if there is an identification of each tangent space with $\mathbb{H} \oplus \mathbb{H}$ taking the $\Phi$ to $\Phi_{0}$. Via such an identification, one may pull back the metric $g_{0}$ to each tangent space giving a well-defined Riemannian metric on $M$. The Spin(7) structure is integrable if the 4form is parallel with respect to the Levi-Civita connection. See [Br1] for a proof of the fact that the Spin(7) structure is integrable if and only if the 4-form is closed. Let us call such manifolds, Spin (7) manifolds or say that $M$ has a $S p i n(7)$ structure.

Let $M^{8}$ be a $\operatorname{Spin}(7)$ manifold with defining 4 -form $\Phi$. We define the Spin (7) coframe bundle $\mathcal{F}(M)$ by letting the fiber $\mathcal{F}_{p}$, for a point p in $M$, be the set of linear maps $u: T_{p}(M) \rightarrow \mathbb{R}^{8}$ where $u^{*}\left(\Phi_{0}\right)=\Phi$. Since $\Phi$ is nondegenerate there is one such map, so by the definition of $\operatorname{Spin}(7)$, there will be a $\operatorname{Spin}(7)$ worth of such maps, and we can give $\mathcal{F}$ a right $\operatorname{Spin}(7)$ action, making $\mathcal{F}$ a principal Spin (7) bundle. Let $\omega$ be the canonical $\mathbb{R}^{8}$ valued form on $\mathcal{F}(M)$, i.e., $\omega=u \circ \pi_{*}$. The expression for $\Phi$ (again pulled up to $\mathcal{F}(M)$ using the projection) is

$$
\begin{aligned}
\Phi= & \omega^{1234}+\left(\omega^{12}-\omega^{34}\right) \wedge\left(\omega^{56}-\omega^{78}\right) \\
& +\left(\omega^{13}+\omega^{24}\right) \wedge\left(\omega^{57}+\omega^{68}\right) \\
& +\left(\omega^{14}-\omega^{23}\right) \wedge\left(\omega^{58}-\omega^{67}\right)+\omega^{5678} .
\end{aligned}
$$

A four dimensional submanifold $X$ of $M$ is said to be Cayley if the tangent space at each point is a Cayley plane. Thus, the form $\Phi$ will restrict 
to be the volume form of $X$. Given such a submanifold we restrict the Spin (7) coframe bundle of $M$ to $X$ and the let $\mathcal{F}^{(1)}(X)$ denote the first adapted coframe bundle where the $\eta_{a}$ 's restrict to zero. This will be a right principal $H$ bundle where $H$ is defined in the previous proposition.

In order to study the deformation theory of Cayley submanifolds, we need the following characterization: A Spin (7) manifold has a natural seven dimensional vector bundle, $E=\mathcal{F}(M) \times{ }_{\rho} \mathbb{R}^{7}$, where $\rho$ is the vector representation of $\operatorname{Spin}(7)$ on $\mathbb{R}^{7}$ defined by pulling back the standard representation of $S O(7)$ on $\mathbb{R}^{7}$. A $S p i n(7)$ manifold carries a 4 -fold cross product with values in the vector bundle $E$. For $V_{1}, V_{2}, V_{3}, V_{4} \in T_{p}(M)$ then

$$
\times:\left(V_{1}, V_{2}, V_{3}, V_{4}\right) \mapsto V_{1} \times V_{2} \times V_{3} \times V_{4} \in E_{p} .
$$

This cross product may be thought of as a 4 -form $\tau \in \Omega^{4}(M, E)$. We have the following:

Proposition 6.2. A four dimensional submanifold of a Spin(7) manifold is Cayley if and only if the form $\tau$ restricts to the submanifold to be zero.

Proof. See [HL].

One can show that the form $\tau$ has components

$$
\left(\begin{array}{c}
\left(\omega^{14}-\omega^{23}\right) \wedge\left(\omega^{57}+\omega^{68}\right)-\left(\omega^{13}+\omega^{24}\right) \wedge\left(\omega^{58}-\omega^{67}\right) \\
\left(\omega^{12}-\omega^{34}\right) \wedge\left(\omega^{58}-\omega^{67}\right)-\left(\omega^{14}-\omega^{23}\right) \wedge\left(\omega^{56}-\omega^{78}\right) \\
\left(\omega^{13}+\omega^{24}\right) \wedge\left(\omega^{56}-\omega^{78}\right)-\left(\omega^{12}-\omega^{34}\right) \wedge\left(\omega^{57}+\omega^{68}\right) \\
\omega^{2345}-\omega^{1346}+\omega^{1247}-\omega^{1238}-\omega^{1678}+\omega^{2578}-\omega^{3568}+\omega^{4567} \\
\omega^{2346}+\omega^{1345}+\omega^{1248}+\omega^{1237}-\omega^{2678}-\omega^{1578}-\omega^{4568}-\omega^{3567} \\
\omega^{2347}+\omega^{1348}-\omega^{1245}-\omega^{1236}-\omega^{3678}-\omega^{4578}+\omega^{1568}+\omega^{2567} \\
\omega^{2348}-\omega^{1347}-\omega^{1246}+\omega^{1235}-\omega^{4567}+\omega^{3578}+\omega^{2568}-\omega^{1567}
\end{array}\right)
$$

We again need several facts from the theory of spinors, see [LM].

(1) Not all four manifolds have a spin structure, but, given a spin structure on the Cayley submanifold $X$, then this gives rise to $S p(1) \times S p(1) \times$ $S p(1)$ principal bundle $\mathcal{P}$ which double covers the bundle $\mathcal{F}^{(1)}(X)$. It is convenient to assume this exists, but none of the constructions depend on it. If $\mathcal{P}$ exists then various vector bundles correspond to representations of $S p(1) \times S p(1) \times S p(1)$, and sections correspond to equivariant maps from $\mathcal{P}$ to the representation space. For example: 
(2) Tangent vectors correspond to maps $V: \mathcal{P} \rightarrow \mathbb{H}$ such that $R_{(p, q, r)}^{*}(V)=\bar{p} V q$.

(3) Normal vectors correspond to maps $V: \mathcal{P} \rightarrow \mathbb{H}$ such that $R_{(p, q, r)}^{*}(V)=$ $\bar{r} V q$.

(4) Anti-self dual 2-forms correspond to maps $A: \mathcal{P} \rightarrow \operatorname{Im} \mathbb{H}$ such that $R_{(p, q, r)}^{*}(A)=\bar{r} A q$.

(5) Positive spinors (sections of $\mathbb{S}_{+}$) correspond to maps $S: \mathcal{P} \rightarrow \mathbb{H}$ such that $R_{(p, q, r)}^{*}(S)=\bar{p} S$, and negative spinors correspond to maps $S$ : $\mathcal{P} \rightarrow \mathbb{H}$ such that $R_{(p, q, r)}^{*}(S)=S q$.

(6) The bundle $E$ on $\mathrm{M}$ arises from a representation of $\operatorname{Spin}(7)$ that when restricted to the subgroup $H$ is given as follows. Writing $\mathbb{R}^{7}$ as $\mathbb{H} \oplus$ $\operatorname{Im} \mathbb{H}$ then $[p, q, r] \in H$ sends $(x, y) \in \mathbb{H} \oplus \operatorname{Im} \mathbb{H}$ to $(\bar{q} x q, \bar{r} y p)$. This may be seen by noting that the form $\tau$ is Spin (7) invariant and so is invariant for the subgroup $H$, and the described action of is precisely the action needed for the invariance under $H$. In particular, the bundle $E$ decomposes naturally when restricted to $X$ into a rank three bundle $E_{1}$ and a rank four bundle $E_{2}$.

(7) If $F$ denotes the bundle whose sections correspond to maps $V: \mathcal{P} \rightarrow \mathbb{H}$ such that $R_{(p, q, r)}^{*}(V)=\bar{r} V$, then the bundle $E$ restricted to $X$ may be identified with $\Lambda_{-}^{2} \oplus \mathbb{S}_{+} \otimes F$, and the normal bundle may be identified with $\mathbb{S}_{-} \otimes F$.

(8) The (twisted) Dirac operator will map sections of $\mathbb{S}_{-} \otimes F$ to sections of $\mathbb{S}_{+} \otimes F$. Thinking of these sections as quaternion valued functions, the Dirac operator is just $\nabla_{1}+i \nabla_{2}+j \nabla_{3}+k \nabla_{4}$, or as a matrix,

$$
\not D=\left(\begin{array}{rrrr}
\nabla_{1} & -\nabla_{2} & -\nabla_{3} & -\nabla_{4} \\
\nabla_{2} & \nabla_{1} & \nabla_{4} & -\nabla_{3} \\
\nabla_{3} & -\nabla_{4} & \nabla_{1} & \nabla_{2} \\
\nabla_{4} & -\nabla_{3} & -\nabla_{2} & \nabla_{1}
\end{array}\right) .
$$

In particlular, the normal vector field $V=V^{a} \frac{\partial}{\partial \omega^{a}}$ is identified with the spinor $V^{5}+i V^{6}+j V^{7}+k V^{8}$, and

$$
\begin{aligned}
\not D(V)= & \left(V_{1}^{5}-V_{2}^{6}-V_{3}^{7}-V_{4}^{8}\right)+i\left(V_{2}^{5}-V_{2}^{6}+V_{4}^{7}-V_{3}^{8}\right) \\
& +j\left(V_{3}^{5}+V_{4}^{6}-V_{1}^{7}+V_{2}^{8}\right)+k\left(V_{4}^{5}-V_{3}^{6}-V_{2}^{7}-V_{1}^{8}\right)
\end{aligned}
$$


With these preliminaries, we may state the following

Theorem 6.3. Under the correspondence of normal vector fields with twisted negative spinors, the Zariski tangent space to Cayley submanifolds at a given Cayley submanifold $f: X \rightarrow M$ is the space of harmonic twisted spinors on $X$, that is, the kernel of the twisted Dirac operator. In other words, the necessary condition on a normal vector field that it be a deformation vector field to a family of Cayley submanifolds is that should correspond to a harmonic spinor in the above isomorphism $N(X) \cong \mathbb{S}_{-} \otimes F$.

Remark. The Atiyah-Singer index theorem gives the index of the twisted Dirac operator $\not \supset: \mathbb{S}_{-} \otimes F \rightarrow \mathbb{S}_{+} \otimes F$ as the integral over $X$ of the degree four piece of

$$
\hat{A}(X) \operatorname{ch}(F)=\left(1+\frac{1}{24} p_{1}(X)+\ldots\right)\left(2+c_{2}(F)+\ldots\right) .
$$

Thus

$$
\text { ind }(\not D)=\int_{X} \frac{1}{12} p_{1}(X)+c_{2}(F)=\frac{1}{4} \sigma(X)+c_{2}(F) \text {. }
$$

Unfortunately, as in the associative case, knowing the index gives no control on the size of the kernel.

Proof of 6.3. We define the nonlinear map $F$ on a neighborhood $U$ of $0 \in \Gamma(N(X))$, i.e., small normal vector fields. $F(V)$ is defined to be the pull back of $\tau$ using the map $\exp _{V} . F$ takes values in

$$
\Omega^{4}(X, f *(E))=\Omega^{3}\left(X, \Lambda_{-}^{2}\right) \oplus \Omega^{3}\left(X, \mathbb{S}_{+} \otimes F\right) .
$$

By Proposition $6.2, F^{-1}(0)$ correspond to nearby associative submanifolds. As in the previous sections, we compute:

$$
\begin{aligned}
F^{\prime}(0)(V) & =\left.\frac{\partial}{\partial t} F(t V)\right|_{t=0} \\
& =\left.\mathcal{L}_{V}(\tau)\right|_{X} \\
& =(V\lrcorner d \tau+d(V\lrcorner \tau))\left.\right|_{X} \\
& =d(V\lrcorner \tau)\left.\right|_{X} .
\end{aligned}
$$


From (6.10), we find

$$
F^{\prime}(0)(V)=\left(\begin{array}{c}
0 \\
0 \\
0 \\
V_{11}-V_{22}-V_{33}-V_{44} \\
V_{12}-V_{22}+V_{34}-V_{43} \\
V_{13}+V_{24}-V_{31}+V_{42} \\
V_{14}-V_{23}-V_{32}-V_{41}
\end{array}\right) \omega^{1234}
$$

Thus comparing (6.12) and (6.13), we see that the infinitesimal associative deformations (normal vector fields in the kernel of $F^{\prime}(0)$ ) are in one to one correspondence with harmonic spinors.

Theorem 6.4. Given a family of submanifolds $X_{t}$ with $X_{0}$ Cayley, then setting $V$ to be the deformation vector field (again assumed normal) then

$$
\left.\frac{d^{2}}{d t^{2}} \operatorname{vol}\left(X_{t}\right)\right|_{t=0}=2 \int_{X}\langle\not D(V), \not D(V)\rangle d v^{2} l_{0} .
$$

Corollary 6.5. Under the identification of the normal bundle with the twisted spin bundle the Jacobi operator is identified with the Dirac Laplacian $\not D^{2}$.

As in the the associative case and because the kernel of $\not D$ is the same as the kernel of $\not D^{2}$ on a compact manifold, we reduce the integrability question of Jacobi fields to the above (difficult) integrability problem.

Proof of the Theorem 6.4. One merely calculates from the expression (6.8) of $\Phi$ :

$$
\begin{aligned}
\mathcal{Q}_{\Phi}(\nabla V)=2 & \left(V_{3}^{5} V_{4}^{6}-V_{4}^{5} V_{3}^{6}-V_{3}^{7} V_{4}^{8}+V_{4}^{7} V_{3}^{8}-V_{1}^{5} V_{2}^{6}+V_{2}^{5} V_{1}^{6}\right. \\
& +V_{1}^{7} V_{2}^{8}-V_{2}^{7} V_{1}^{8}+V_{4}^{5} V_{2}^{7}-V_{2}^{5} V_{4}^{7}+V_{4}^{6} V_{2}^{8}-V_{2}^{6} V_{4}^{8} \\
& +V_{3}^{5} V_{1}^{7}-V_{1}^{5} V_{3}^{7}+V_{3}^{6} V_{1}^{8}-V_{1}^{6} V_{3}^{8}-V_{3}^{5} V_{2}^{8}+V_{2}^{5} V_{3}^{8} \\
& \left.+V_{3}^{6} V_{2}^{7}-V_{2}^{6} V_{3}^{7}+V_{4}^{5} V_{1}^{8}-V_{1}^{5} V_{4}^{8}-V_{4}^{6} V_{1}^{7}+V_{1}^{6} V_{4}^{7}\right)
\end{aligned}
$$

and then show

$$
\|\nabla V\|^{2}-\mathcal{Q}_{\Phi}(\nabla V)=\langle\not \supset(V), \not \supset(V)\rangle
$$

and the theorem follows from (2.13). 
Examples. Again, there are flat examples given by Cayley tori in Spin (7) tori, but, more importantly, Bryant and Salamon ([BS]) construct a metric with holonomy Spin (7) on (an open set of) the total space of the negative spin bundle $\mathbb{S}_{-}$of a spinnable self-dual Einstein four manifold $X$. From their formulae, the zero section (which we identify with $X$ ) is Cayley. It is clear that the normal bundle to the zero section is just the spin bundle $\mathbb{S}_{-}$, itself. Hence, the bundle $F$ defined in the seventh remark is trivial, and the Zariski tangent space at $X$ to the moduli of Cayley submanifolds is isomorphic to the harmonic (negative) spinors rather than harmonic twisted spinors.

There are three cases, the scalar curvature $S$ of $X$ is positive, negative, or zero. In the case $S>0, X$ is necessarily the four sphere by a result of Hitchin $[\mathrm{H}]$, and there are no harmonic spinors by a vanishing theorem of Lichnerowicz, [Li]. Hence, the zero section is rigid. In the case $S=0$, the bundle is actually trivial, and the metric constructed in [BS] is just the product metric of the base four manifold with the flat metric on $\mathbb{R}^{4}$. The zero section is deformable in the obvious four dimensional family. In the case $S<0$, it may be that the zero section is deformable as a Cayley submanifold.

\section{Conclusion.}

Several interesting problems remain. We list a few:

(1) First of all, we have shown that the moduli space of either special Lagrangian submanifolds of Calabi-Yau manifolds or coassociative submanifolds of $G_{2}$ manifolds carry riemannian structures. Now, a differential geometer when coming upon a Riemmanian manifold, first calculates the Riemmanian curvature. Do the curvatures of these moduli spaces, satisfy some nice relations? Constant curvature, Einstein, ... ?

(2) The moduli space of special Lagrangian submanifolds carries an nform. Is it closed?, co-closed?, even relevant? It could zero just by dimension reasons.

(3) There are questions on global problems of the moduli. We have already seen that the moduli space of special Lagrangian (=complex) tori in elliptic $K 3$ surfaces is essentially non-compact due to the degenerate leaves. Can one somehow control the infinity of the moduli space? Is there some sort of weak compactness?

These questions probably would entail studying the possible singularities of calibrated submanifolds in flat space. This seems difficult. One 
first studies conical calibrated submanifolds. One studies these cones by first intersecting with a sphere and thereby obtain submanifolds of the sphere (of dimension one less) with special properties. For example, conical associative submanifolds give rise to Riemann surfaces in the six-sphere that are holomorphic with respect to the standard almost complex structure on the six-sphere. Bryant in [Br3] has shown that arbitrarily high genus can occur.

(4) The most interesting question is that of trying to turn the problem around: From knowledge of the submanifolds, try to pin down the ambient manifold. The guiding example is that of elliptic K3's. Can one repeat the picture for other manifolds? Two examples:

First, there is a natural generalisation of elliptic $K 3$ 's to arbitrary dimension: The subclass of Calabi-Yau manifolds of dimension $2 n$, with $n$-dimensional real tori that are special Lagrangian. By the results of the paper, the special Lagrangian tori will move in an $n$-dimensional family. So at least locally, one expects the special Lagrangian tori will foliate the ambient manifold. Whether the ambient mainifold is globally foliated (again allowing for some degenerate leaves) is a difficult question. For elliptic $K 3$ 's one has this result by algebro-geometric arguments.

The second would be is to try to build a nontrivial $G_{2}$ manifold foliated by coassociative $\overline{K 3}$ 's. Given one $\overline{K 3}$, then it will move in a three dimensional family. Again, one must probably allow for degenerations. Kodaira classified the degenerations of complex families of elliptic curves over the disk. Can one classify the degenerations of coassociative $\overline{K 3}$ 's over the three-ball where the total space has a $G_{2}$ structure?

These and other questions will be left for future investigation.

\section{References.}

[Br1] R. Bryant,Metrics with Exceptional Holonomy, Ann. of Math. 126 (1987), 525-576.

[Br2] R. Bryant, Minimal Lagrangian Submanifolds of Kähler-Einstein Manifolds, Lecture Notes in Mathematics, 1255 (1985).

[Br3] R. Bryant, Submanifolds and Special Structures on the Octonions, J. of Diff. Geom. 17 (1982), 185-232. 
[BS] R. Bryant and S. Salamon, On the Construction of Some Complete Metrics with Exceptional Holonomy, Duke Math. J. 58 (1989), 829-850.

[Ch1] S. S. Chern, Minimal Submanifolds in a Riemannian Manifold, University of Kansas Tech. Report 19 (1968), (See also the Collected works of Chern vol. 4, Springer-Verlag, 1989).

[Ch2] S. S. Chern, Complex Manifolds without Potential Theory, Springer-Verlag, (1979).

[G] A. Grothendieck, Techniques de Construction et Theoremes d'existence en Geometrie Algebrique IV: Les Schemas de Hilbert, Seminaire Bourbaki, 221 (1960/61).

[Gr] P. Griffiths, Exterior Differential Systems and the Calculus of Variations, Progress in Mathematics, 25, Birkhauser, Boston, (1983).

[GH] P. Griffiths and J. Harris, Principles of Algebraic Geometry, Wiley Interscience, (1978).

[H] F. R. Harvey, Spinors and Calibrations, Academic Press, (1990).

[HL] R. Harvey and H. B. Lawson, Jr., Calibrated Geometries, Acta Math. 148 (1982), 47-157.

[Hi] N. Hitchin, Kahlerian Twistor Spaces, Proc. London Math. Soc. 43 (1981), 133-150.

[K1] K. Kodaira, A Theorem of Completeness of Characteristic Systems for Analytic Families of Compact Submanifolds of Complex Manifolds, Ann. of Math. 75 (1962), 146-162.

[K2] K. Kodaira, On compact complex analytic surfaces, Ann. of Math. 71 (1960), 111-152; II, Ann. of Math. 77 (1963), 563-626; III, Ann. of Math. 78 (1963), $1-40$.

[L] H. B. Lawson, Jr., Minimal Varieties in Real and Complex Geometry, Séminaire de Mathématiques Supérieures Université de Montréal, (1974).

[LM] H. B. Lawson and M.-L. Michelson, Spin Geometry, Princeton University Press, (1989).

[Li] A. Lichnerowicz, Spineurs Harmoniques, C. R. Acad. Sci. Paris Ser A-B, 257 (1963), 7-9.

[S] J. Simons, Minimal Varieties of Riemannian Manifolds, Ann. of Math. 88 (1968), 47-157. 
[Y] S. T. Yau, On the Ricci Curvature of a Compact Kähler Manifold and the Complex Monge-Ampere Equation I, Comm. of Pure and Applied Math. 31 (1978), 339-411.

ReCeived September 3, 1996.

5601 TETON

COLUMBIA, MO 65201

E-mail address: surgrm@showme.medicine.edu 